\title{
Searches for rare or forbidden semileptonic charm decays
}

J. P. Lees, ${ }^{1}$ V. Poireau, ${ }^{1}$ V. Tisserand, ${ }^{1}$ J. Garra Tico, ${ }^{2}$ E. Grauges,${ }^{2}$ M. Martinelli, ${ }^{3 a, 3 b}$ D. A. Milanes, ${ }^{3 a}$ A. Palano, ${ }^{3 a, 3 b}$ M. Pappagallo, ${ }^{3 a, 3 b}$ G. Eigen, ${ }^{4}$ B. Stugu, ${ }^{4}$ L. Sun, ${ }^{4}$ D. N. Brown,${ }^{5}$ L. T. Kerth, ${ }^{5}$ Yu. G. Kolomensky, ${ }^{5}$ G. Lynch, ${ }^{5}$ H. Koch, ${ }^{6}$ T. Schroeder, ${ }^{6}$ D. J. Asgeirsson, ${ }^{7}$ C. Hearty,${ }^{7}$ T. S. Mattison, ${ }^{7}$ J. A. McKenna, ${ }^{7}$ A. Khan, ${ }^{8}$ V. E. Blinov, ${ }^{9}$ A. R. Buzykaev, ${ }^{9}$ V. P. Druzhinin, ${ }^{9}$ V. B. Golubev, ${ }^{9}$ E. A. Kravchenko, ${ }^{9}$ A. P. Onuchin, ${ }^{9}$ S. I. Serednyakov, ${ }^{9}$ Yu. I. Skovpen, ${ }^{9}$ E. P. Solodov, ${ }^{9}$

K. Yu. Todyshev, ${ }^{9}$ A. N. Yushkov, ${ }^{9}$ M. Bondioli, ${ }^{10}$ S. Curry, ${ }^{10}$ D. Kirkby,${ }^{10}$ A. J. Lankford, ${ }^{10}$ M. Mandelkern, ${ }^{10}$

D. P. Stoker, ${ }^{10}$ H. Atmacan, ${ }^{11}$ J. W. Gary, ${ }^{11}$ F. Liu, ${ }^{11}$ O. Long, ${ }^{11}$ G. M. Vitug,${ }^{11}$ C. Campagnari, ${ }^{12}$ T. M. Hong, ${ }^{12}$

D. Kovalskyi, ${ }^{12}$ J. D. Richman, ${ }^{12}$ C. A. West, ${ }^{12}$ A. M. Eisner, ${ }^{13}$ J. Kroseberg, ${ }^{13}$ W. S. Lockman, ${ }^{13}$ A. J. Martinez, ${ }^{13}$

T. Schalk, ${ }^{13}$ B. A. Schumm, ${ }^{13}$ A. Seiden, ${ }^{13}$ C. H. Cheng, ${ }^{14}$ D. A. Doll, ${ }^{14}$ B. Echenard,${ }^{14}$ K. T. Flood, ${ }^{14}$ D. G. Hitlin, ${ }^{14}$

P. Ongmongkolkul, ${ }^{14}$ F. C. Porter ${ }^{14}$ A. Y. Rakitin, ${ }^{14}$ R. Andreassen, ${ }^{15}$ M. S. Dubrovin, ${ }^{15}$ B. T. Meadows,${ }^{15}$

M. D. Sokoloff, ${ }^{15}$ P. C. Bloom, ${ }^{16}$ W. T. Ford ${ }^{16}$ A. Gaz, ${ }^{16}$ M. Nagel,${ }^{16}$ U. Nauenberg, ${ }^{16}$ J. G. Smith, ${ }^{16}$ S. R. Wagner,${ }^{16}$

R. Ayad, ${ }^{17}, *$ W. H. Toki, ${ }^{17}$ B. Spaan,${ }^{18}$ M. J. Kobel, ${ }^{19}$ K. R. Schubert, ${ }^{19}$ R. Schwierz, ${ }^{19}$ D. Bernard, ${ }^{20}$ M. Verderi,${ }^{20}$

P. J. Clark ${ }^{21}$ S. Playfer, ${ }^{21}$ D. Bettoni, ${ }^{22 a}$ C. Bozzi, ${ }^{22 a}$ R. Calabrese, ${ }^{22 a, 22 b}$ G. Cibinetto, ${ }^{22 a, 22 b}$ E. Fioravanti, ${ }^{22 a, 22 b}$

I. Garzia, ${ }^{22 a, 22 b}$ E. Luppi, ${ }^{22 a, 22 b}$ M. Munerato, ${ }^{22 a, 22 b}$ M. Negrini, ${ }^{22 a, 22 b}$ L. Piemontese, ${ }^{22 a}$ R. Baldini-Ferroli, ${ }^{23}$

A. Calcaterra, ${ }^{23}$ R. de Sangro, ${ }^{23}$ G. Finocchiaro, ${ }^{23}$ M. Nicolaci, ${ }^{23}$ P. Patteri, ${ }^{23}$ I. M. Peruzzii, ${ }^{23, \dagger}$ M. Piccolo, ${ }^{23}$ M. Rama, ${ }^{23}$ A. Zallo, ${ }^{23}$ R. Contri, ${ }^{24 a, 24 b}$ E. Guido, ${ }^{24 a, 24 b}$ M. Lo Vetere, ${ }^{24 a, 24 b}$ M. R. Monge, ${ }^{24 a, 24 b}$ S. Passaggio, ${ }^{24 a}$ C. Patrignani, ${ }^{24 a, 24 b}$ E. Robutti, ${ }^{24 a}$ B. Bhuyan, ${ }^{25}$ V. Prasad, ${ }^{25}$ C. L. Lee, ${ }^{26}$ M. Morii, ${ }^{26}$ A. J. Edwards, ${ }^{27}$ A. Adametz, ${ }^{28}$ J. Marks, ${ }^{28}$ U. Uwer, ${ }^{28}$ F. U. Bernlochner, ${ }^{29}$ M. Ebert, ${ }^{29}$ H. M. Lacker, ${ }^{29}$ T. Lueck ${ }^{29}$ P. D. Dauncey, ${ }^{30}$ M. Tibbetts,${ }^{30}$ P. K. Behera ${ }^{31}$ U. Mallik,${ }^{31}$ C. Chen, ${ }^{32}$ J. Cochran, ${ }^{32}$ H. B. Crawley, ${ }^{32}$ W. T. Meyer, ${ }^{32}$ S. Prell, ${ }^{32}$ E. I. Rosenberg, ${ }^{32}$ A. E. Rubin, ${ }^{32}$ A. V. Gritsan, ${ }^{33}$ Z. J. Guo, ${ }^{33}$ N. Arnaud, ${ }^{34}$ M. Davier, ${ }^{34}$ G. Grosdidier ${ }^{34}$ F. Le Diberder,${ }^{34}$ A. M. Lutz ${ }^{34}$ B. Malaescu, ${ }^{34}$ P. Roudeau, ${ }^{34}$ M. H. Schune, ${ }^{34}$ A. Stocchi, ${ }^{34}$ G. Wormser,${ }^{34}$ D. J. Lange, ${ }^{35}$ D. M. Wright, ${ }^{35}$ I. Bingham, ${ }^{36}$ C. A. Chavez, ${ }^{36}$ J. P. Coleman, ${ }^{36}$

J. R. Fry, ${ }^{36}$ E. Gabathuler, ${ }^{36}$ D. E. Hutchcroft,${ }^{36}$ D. J. Payne, ${ }^{36}$ C. Touramanis, ${ }^{36}$ A. J. Bevan, ${ }^{37}$ F. Di Lodovico, ${ }^{37}$ R. Sacco, ${ }^{37}$ M. Sigamani, ${ }^{37}$ G. Cowan, ${ }^{38}$ S. Paramesvaran, ${ }^{38}$ D. N. Brown, ${ }^{39}$ C. L. Davis, ${ }^{39}$ A. G. Denig, ${ }^{40}$ M. Fritsch, ${ }^{40}$ W. Gradl ${ }^{40}$ A. Hafner,${ }^{40}$ E. Prencipe,${ }^{40}$ K. E. Alwyn, ${ }^{41}$ D. Bailey, ${ }^{41}$ R. J. Barlow, ${ }^{41}$ G. Jackson, ${ }^{41}$ G. D. Lafferty, ${ }^{41}$ R. Cenci ${ }^{42}$ B. Hamilton, ${ }^{42}$ A. Jawahery, ${ }^{42}$ D. A. Roberts, ${ }^{42}$ G. Simi, ${ }^{42}$ C. Dallapiccola, ${ }^{43}$ R. Cowan, ${ }^{44}$ D. Dujmic,${ }^{44}$ G. Sciolla, ${ }^{44}$ D. Lindemann, ${ }^{45}$ P. M. Patel, ${ }^{45}$ S. H. Robertson, ${ }^{45}$ M. Schram,${ }^{45}$ P. Biassoni, ${ }^{46 a, 46 b}$ A. Lazzaro, ${ }^{46 a, 46 b}$

V. Lombardo, ${ }^{46 \mathrm{a}}$ F. Palombo, ${ }^{46 a, 46 \mathrm{~b}}$ S. Stracka, ${ }^{46 a, 46 \mathrm{~b}}$ L. Cremaldi, ${ }^{47}$ R. Godang, ${ }^{47, \$}$ R. Kroeger ${ }^{47}$ P. Sonnek, ${ }^{47}$ D. J. Summers, ${ }^{47}$ X. Nguyen, ${ }^{48}$ P. Taras, ${ }^{48}$ G. De Nardo,${ }^{49 a, 49 b}$ D. Monorchio, ${ }^{49 a, 49 b}$ G. Onorato, ${ }^{49 a, 49 b}$ C. Sciacca, ${ }^{49 a, 49 b}$ G. Raven ${ }^{50}$ H. L. Snoek, ${ }^{50}$ C. P. Jessop,${ }^{51}$ K. J. Knoepfel, ${ }^{51}$ J. M. LoSecco, ${ }^{51}$ W. F. Wang, ${ }^{51}$ K. Honscheid ${ }^{52}$ R. Kass,${ }^{52}$ J. Brau, ${ }^{53}$ R. Frey, ${ }^{53}$ N. B. Sinev,${ }^{53}$ D. Strom, ${ }^{53}$ E. Torrence, ${ }^{53}$ E. Feltresi, ${ }^{54 a, 54 b}$ N. Gagliardi, ${ }^{54 a, 54 b}$ M. Margoni, ${ }^{54 a, 54 b}$ M. Morandin, ${ }^{54 a}$ M. Posocco,${ }^{54 a}$ M. Rotondo, ${ }^{54 a}$ F. Simonetto, ${ }^{54 a, 54 b}$ R. Stroili, ${ }^{54 a, 54 b}$ E. Ben-Haim, ${ }^{55}$ M. Bomben, ${ }^{55}$ G. R. Bonneaud, ${ }^{55}$ H. Briand, ${ }^{55}$ G. Calderini, ${ }^{55}$ J. Chauveau, ${ }^{55}$ O. Hamon,${ }^{55}$ Ph. Leruste, ${ }^{55}$ G. Marchiori, ${ }^{55}$ J. Ocariz ${ }^{55}$ S. Sitt, ${ }^{55}$ M. Biasini, ${ }^{56 a, 56 b}$ E. Manoni, ${ }^{56 a, 56 b}$ S. Pacetti, ${ }^{56 a, 56 b}$ A. Rossi, ${ }^{56 a, 56 b}$ C. Angelini, ${ }^{57 a, 57 b}$ G. Batignani, ${ }^{57 a, 57 b}$ S. Bettarini, ${ }^{57 a, 57 b}$ M. Carpinelli, ${ }^{57 a, 57 b},{ }^{8}$ G. Casarosa, ${ }^{57 a, 57 b}$ A. Cervelli, ${ }^{57 a, 57 b}$ F. Forti, ${ }^{57 a, 57 b}$ M. A. Giorgi, ${ }^{57 a, 57 b}$ A. Lusiani, ${ }^{57 a, 57 c}$ N. Neri, ${ }^{57 a, 57 b}$ B. Oberhof, ${ }^{57 a, 57 b}$ E. Paoloni, ${ }^{57 a, 57 b}$ A. Perez, ${ }^{57 a}$ G. Rizzo, ${ }^{57 a, 57 b}$ J. J. Walsh, ${ }^{57 a}$ D. Lopes Pegna, ${ }^{58}$ C. Lu, ${ }^{58}$ J. Olsen, ${ }^{58}$ A. J. S. Smith, ${ }^{58}$ A. V. Telnov, ${ }^{58}$ F. Anulli, ${ }^{59 a}$ G. Cavoto, ${ }^{59 a}$ R. Faccini, ${ }^{59 a, 59 b}$ F. Ferrarotto, ${ }^{59 a}$ F. Ferroni, ${ }^{59 a, 59 b}$ M. Gaspero, ${ }^{59 a, 59 b}$ L. Li Gioi, ${ }^{59 a}$ M. A. Mazzoni,${ }^{59 a}$ G. Piredda, ${ }^{59 a}$ C. Bünger,${ }^{60}$ O. Grünberg, ${ }^{60}$ T. Hartmann, ${ }^{60}$ T. Leddig, ${ }^{60}$ H. Schröder ${ }^{60}$ R. Waldi, ${ }^{60}$ T. Adye, ${ }^{61}$ E. O. Olaiya, ${ }^{61}$ F. F. Wilson,${ }^{61}$

S. Emery, ${ }^{62}$ G. Hamel de Monchenault, ${ }^{62}$ G. Vasseur ${ }^{62}$ Ch. Yèche, ${ }^{62}$ D. Aston, ${ }^{63}$ D. J. Bard,${ }^{63}$ R. Bartoldus, ${ }^{63}$ J. F. Benitez ${ }^{63}$ C. Cartaro, ${ }^{63}$ M. R. Convery, ${ }^{63}$ J. Dorfan, ${ }^{63}$ G. P. Dubois-Felsmann, ${ }^{63}$ W. Dunwoodie, ${ }^{63}$ R. C. Field, ${ }^{63}$ M. Franco Sevilla, ${ }^{63}$ B. G. Fulsom, ${ }^{63}$ A. M. Gabareen ${ }^{63}$ M. T. Graham, ${ }^{63}$ P. Grenier, ${ }^{63}$ C. Hast, ${ }^{63}$ W. R. Innes,${ }^{63}$ M. H. Kelsey, ${ }^{63}$ H. Kim,${ }^{63}$ P. Kim, ${ }^{63}$ M. L. Kocian, ${ }^{63}$ D. W. G. S. Leith ${ }^{63}$ P. Lewis, ${ }^{63}$ S. Li, ${ }^{63}$ B. Lindquist ${ }^{63}$ S. Luitz,${ }^{63}$ V. Luth, ${ }^{63}$ H. L. Lynch, ${ }^{63}$ D. B. MacFarlane, ${ }^{63}$ D. R. Muller, ${ }^{63}$ H. Neal, ${ }^{63}$ S. Nelson, ${ }^{63}$ I. Ofte, ${ }^{63}$ M. Perl, ${ }^{63}$ T. Pulliam, ${ }^{63}$ B. N. Ratcliff, ${ }^{63}$ A. Roodman, ${ }^{63}$ A. A. Salnikov, ${ }^{63}$ V. Santoro, ${ }^{63}$ R. H. Schindler, ${ }^{63}$ A. Snyder, ${ }^{63}$ D. Su, ${ }^{63}$ M. K. Sullivan, ${ }^{63}$ J. Va'vra, ${ }^{63}$ A. P. Wagner, ${ }^{63}$ M. Weaver, ${ }^{63}$ W. J. Wisniewski, ${ }^{63}$ M. Wittgen, ${ }^{63}$ D. H. Wright, ${ }^{63}$ H. W. Wulsin, ${ }^{63}$

A. K. Yarritu, ${ }^{63}$ C. C. Young, ${ }^{63}$ V. Ziegler, ${ }^{63}$ W. Park, ${ }^{64}$ M. V. Purohit,${ }^{64}$ R. M. White,${ }^{64}$ J. R. Wilson, ${ }^{64}$ A. Randle-Conde, ${ }^{65}$ S. J. Sekula, ${ }^{65}$ M. Bellis, ${ }^{66}$ P. R. Burchat, ${ }^{66}$ T. S. Miyashita ${ }^{66}$ B. A. Petersen, ${ }^{66}$ M. S. Alam, ${ }^{67}$ J. A. Ernst,${ }^{67}$

R. Gorodeisky, ${ }^{68}$ N. Guttman, ${ }^{68}$ D. R. Peimer, ${ }^{68}$ A. Soffer, ${ }^{68}$ P. Lund, ${ }^{69}$ S. M. Spanier,${ }^{69}$ R. Eckmann, ${ }^{70}$ J. L. Ritchie, ${ }^{70}$ A. M. Ruland, ${ }^{70}$ C. J. Schilling, ${ }^{70}$ R. F. Schwitters, ${ }^{70}$ B. C. Wray, ${ }^{70}$ J. M. Izen, ${ }^{71}$ X. C. Lou, ${ }^{71}$ F. Bianchi, ${ }^{72 a, 72 b}$ D. Gamba, ${ }^{72 a, 72 b}$ L. Lanceri, ${ }^{73 a, 73 b}$ L. Vitale, ${ }^{73 a, 73 b}$ N. Lopez-March, ${ }^{74}$ F. Martinez-Vidal, ${ }^{74}$ A. Oyanguren, ${ }^{74}$ H. Ahmed, ${ }^{75}$ 
J. Albert, ${ }^{75}$ Sw. Banerjee,${ }^{75}$ H. H.F. Choi, ${ }^{75}$ G. J. King, ${ }^{75}$ R. Kowalewski, ${ }^{75}$ M. J. Lewczuk, ${ }^{75}$ C. Lindsay, ${ }^{75}$ I. M. Nugent, ${ }^{75}$ J. M. Roney,${ }^{75}$ R. J. Sobie, ${ }^{75}$ T. J. Gershon, ${ }^{76}$ P. F. Harrison, ${ }^{76}$ T. E. Latham, ${ }^{76}$ E. M. T. Puccio, ${ }^{76}$ H. R. Band,${ }^{77}$ S. Dasu, ${ }^{77}$ Y. Pan, ${ }^{77}$ R. Prepost,${ }^{77}$ C. O. Vuosalo, ${ }^{77}$ and S. L. Wu ${ }^{77}$

(BABAR Collaboration)

\footnotetext{
${ }^{1}$ Laboratoire d'Annecy-le-Vieux de Physique des Particules (LAPP), Université de Savoie, CNRS/IN2P3, F-74941 Annecy-Le-Vieux, France

${ }^{2}$ Universitat de Barcelona, Facultat de Fisica, Departament ECM, E-08028 Barcelona, Spain

${ }^{3 a}$ INFN Sezione di Bari, I-70126 Bari, Italy

${ }^{3 \mathrm{~b}}$ Dipartimento di Fisica, Università di Bari, I-70126 Bari, Italy

${ }^{4}$ University of Bergen, Institute of Physics, N-5007 Bergen, Norway

${ }^{5}$ Lawrence Berkeley National Laboratory and University of California, Berkeley, California 94720, USA

${ }^{6}$ Ruhr Universität Bochum, Institut für Experimentalphysik 1, D-44780 Bochum, Germany

${ }^{7}$ University of British Columbia, Vancouver, British Columbia, Canada V6T 1Z1

${ }^{8}$ Brunel University, Uxbridge, Middlesex UB8 3PH, United Kingdom

${ }^{9}$ Budker Institute of Nuclear Physics, Novosibirsk 630090, Russia

${ }^{10}$ University of California at Irvine, Irvine, California 92697, USA

${ }^{11}$ University of California at Riverside, Riverside, California 92521, USA

${ }^{12}$ University of California at Santa Barbara, Santa Barbara, California 93106, USA

${ }^{13}$ University of California at Santa Cruz, Institute for Particle Physics, Santa Cruz, California 95064, USA

${ }^{14}$ California Institute of Technology, Pasadena, California 91125, USA

${ }^{15}$ University of Cincinnati, Cincinnati, Ohio 45221, USA

${ }^{16}$ University of Colorado, Boulder, Colorado 80309, USA

${ }^{17}$ Colorado State University, Fort Collins, Colorado 80523, USA

${ }^{18}$ Technische Universität Dortmund, Fakultät Physik, D-44221 Dortmund, Germany

${ }^{19}$ Technische Universität Dresden, Institut für Kern- und Teilchenphysik, D-01062 Dresden, Germany

${ }^{20}$ Laboratoire Leprince-Ringuet, Ecole Polytechnique, CNRS/IN2P3, F-91128 Palaiseau, France

${ }^{21}$ University of Edinburgh, Edinburgh EH9 3JZ, United Kingdom

${ }^{22 a}$ INFN Sezione di Ferrara, I-44100 Ferrara, Italy

${ }^{22 \mathrm{~b}}$ Dipartimento di Fisica, Università di Ferrara, I-44100 Ferrara, Italy

${ }^{23}$ INFN Laboratori Nazionali di Frascati, I-00044 Frascati, Italy

${ }^{24 a}$ INFN Sezione di Genova, I-16146 Genova, Italy

${ }^{24 \mathrm{~b}}$ Dipartimento di Fisica, Università di Genova, I-16146 Genova, Italy

${ }^{25}$ Indian Institute of Technology Guwahati, Guwahati, Assam, 781 039, India

${ }^{26}$ Harvard University, Cambridge, Massachusetts 02138, USA

${ }^{27}$ Harvey Mudd College, Claremont, California 91711, USA

${ }^{28}$ Universität Heidelberg, Physikalisches Institut, Philosophenweg 12, D-69120 Heidelberg, Germany

${ }^{29}$ Humboldt-Universität zu Berlin, Institut für Physik, Newtonstr. 15, D-12489 Berlin, Germany

${ }^{30}$ Imperial College London, London, SW7 2AZ, United Kingdom

${ }^{31}$ University of Iowa, Iowa City, Iowa 52242, USA

${ }^{32}$ Iowa State University, Ames, Iowa 50011-3160, USA

${ }^{33}$ Johns Hopkins University, Baltimore, Maryland 21218, USA

${ }^{34}$ Laboratoire de l'Accélérateur Linéaire, IN2P3/CNRS et Université Paris-Sud 11,

Centre Scientifique d'Orsay, B. P. 34, F-91898 Orsay Cedex, France

${ }^{35}$ Lawrence Livermore National Laboratory, Livermore, California 94550, USA

${ }^{36}$ University of Liverpool, Liverpool L69 7ZE, United Kingdom

${ }^{37}$ Queen Mary, University of London, London, E1 4NS, United Kingdom

${ }^{38}$ University of London, Royal Holloway and Bedford New College, Egham, Surrey TW20 OEX, United Kingdom

${ }^{39}$ University of Louisville, Louisville, Kentucky 40292, USA

${ }^{40}$ Johannes Gutenberg-Universität Mainz, Institut für Kernphysik, D-55099 Mainz, Germany

${ }^{41}$ University of Manchester, Manchester M13 9PL, United Kingdom

${ }^{42}$ University of Maryland, College Park, Maryland 20742, USA

${ }^{43}$ University of Massachusetts, Amherst, Massachusetts 01003, USA

${ }^{44}$ Massachusetts Institute of Technology, Laboratory for Nuclear Science, Cambridge, Massachusetts 02139, USA

${ }^{45}$ McGill University, Montréal, Québec, Canada H3A 2T8

${ }^{46 a}$ INFN Sezione di Milano, I-20133 Milano, Italy

${ }^{46 \mathrm{~b}}$ Dipartimento di Fisica, Università di Milano, I-20133 Milano, Italy

${ }^{47}$ University of Mississippi, University, Mississippi 38677, USA
} 
${ }^{48}$ Université de Montréal, Physique des Particules, Montréal, Québec, Canada H3C 3J7

${ }^{49 a}$ INFN Sezione di Napoli, I-80126 Napoli, Italy

${ }^{49 \mathrm{~b}}$ Dipartimento di Scienze Fisiche, Università di Napoli Federico II, I-80126 Napoli, Italy

${ }^{50}$ NIKHEF, National Institute for Nuclear Physics and High Energy Physics, NL-1009 DB Amsterdam, The Netherlands

${ }^{51}$ University of Notre Dame, Notre Dame, Indiana 46556, USA

${ }^{52}$ Ohio State University, Columbus, Ohio 43210, USA

${ }^{53}$ University of Oregon, Eugene, Oregon 97403, USA

${ }^{54 a}$ INFN Sezione di Padova, I-35131 Padova, Italy

${ }^{54 b}$ Dipartimento di Fisica, Università di Padova, I-35131 Padova, Italy

${ }^{55}$ Laboratoire de Physique Nucléaire et de Hautes Energies, IN2P3/CNRS, Université Pierre et Marie Curie-Paris6,

Université Denis Diderot-Paris7, F-75252 Paris, France

${ }^{56 a}$ INFN Sezione di Perugia, I-06100 Perugia, Italy

${ }^{56 \mathrm{~b}}$ Dipartimento di Fisica, Università di Perugia, I-06100 Perugia, Italy

${ }^{57}$ INFN Sezione di Pisa, I-56127 Pisa, Italy

${ }^{57 b}$ Dipartimento di Fisica, Università di Pisa, I-56127 Pisa, Italy

${ }^{57 \mathrm{c}}$ Scuola Normale Superiore di Pisa, I-56127 Pisa, Italy

${ }^{58}$ Princeton University, Princeton, New Jersey 08544, USA

${ }^{59 a}$ INFN Sezione di Roma, I-00185 Roma, Italy

${ }^{59 \mathrm{~b}}$ Dipartimento di Fisica, Università di Roma La Sapienza, I-00185 Roma, Italy

${ }^{60}$ Universität Rostock, D-18051 Rostock, Germany

${ }^{61}$ Rutherford Appleton Laboratory, Chilton, Didcot, Oxon, OX11 0QX, United Kingdom

${ }^{62}$ CEA, Irfu, SPP, Centre de Saclay, F-91191 Gif-sur-Yvette, France

${ }^{63}$ SLAC National Accelerator Laboratory, Stanford, California 94309, USA

${ }^{64}$ University of South Carolina, Columbia, South Carolina 29208, USA

${ }^{65}$ Southern Methodist University, Dallas, Texas 75275, USA

${ }^{66}$ Stanford University, Stanford, California 94305-4060, USA

${ }^{67}$ State University of New York, Albany, New York 12222, USA

${ }^{68}$ Tel Aviv University, School of Physics and Astronomy, Tel Aviv, 69978, Israel

${ }^{69}$ University of Tennessee, Knoxville, Tennessee 37996, USA

${ }^{70}$ University of Texas at Austin, Austin, Texas 78712, USA

${ }^{71}$ University of Texas at Dallas, Richardson, Texas 75083, USA

${ }^{72 a}$ INFN Sezione di Torino, I-10125 Torino, Italy

${ }^{72 b}$ Dipartimento di Fisica Sperimentale, Università di Torino, I-10125 Torino, Italy

${ }^{73 a}$ INFN Sezione di Trieste, I-34127 Trieste, Italy

${ }^{73 b}$ Dipartimento di Fisica, Università di Trieste, I-34127 Trieste, Italy

${ }^{74}$ IFIC, Universitat de Valencia-CSIC, E-46071 Valencia, Spain

${ }^{75}$ University of Victoria, Victoria, British Columbia, Canada V8W $3 P 6$

${ }^{76}$ Department of Physics, University of Warwick, Coventry CV4 7AL, United Kingdom

${ }^{77}$ University of Wisconsin, Madison, Wisconsin 53706, USA

(Received 26 July 2011; published 28 October 2011)

We present searches for rare or forbidden charm decays of the form $X_{c}^{+} \rightarrow h^{ \pm} \ell^{\mp} \ell^{(1)+}$, where $X_{c}^{+}$is a charm hadron $\left(D^{+}, D_{s}^{+}\right.$, or $\left.\Lambda_{c}^{+}\right), h^{ \pm}$is a pion, kaon, or proton, and $\ell^{(\prime) \pm}$ is an electron or muon. The analysis is based on $384 \mathrm{fb}^{-1}$ of $e^{+} e^{-}$annihilation data collected at or close to the $\mathrm{Y}(4 S)$ resonance with the BABAR detector at the SLAC National Accelerator Laboratory. No significant signal is observed for any of the 35 decay modes that are investigated. We establish $90 \%$ confidence-level upper limits on the branching fractions between $1 \times 10^{-6}$ and $44 \times 10^{-6}$ depending on the channel. In most cases, these results represent either the first limits or significant improvements on existing limits for the decay modes studied.

DOI: 10.1103/PhysRevD.84.072006

PACS numbers: 11.30.Fs, 11.30.Hv, 13.20.Fc, 13.30.Ce

\section{INTRODUCTION}

We present searches for charm hadron decays that are either forbidden or heavily suppressed in the standard model (SM) of particle physics. The decays are of the

\footnotetext{
*Now at Temple University, Philadelphia, PA 19122, USA

${ }^{\dagger}$ Also with Università di Perugia, Dipartimento di Fisica, Perugia, Italy

Now at University of South AL, Mobile, AL 36688, USA

${ }^{\S}$ Also with Università di Sassari, Sassari, Italy
} form $^{1} X_{c}^{+} \rightarrow h^{ \pm} \ell^{\mp} \ell^{(1)+}$, where $X_{c}^{+}$is a charm hadron

\footnotetext{
${ }^{1}$ The inclusion of charge-conjugate decay modes is implied throughout this paper.
} 
$\left(D^{+}, D_{s}^{+}\right.$, or $\left.\Lambda_{c}^{+}\right)$, and $\ell^{(\prime) \pm}$ is an electron or muon. For $D^{+}$ and $D_{s}^{+}$modes, $h^{ \pm}$can be a pion or kaon, while for $\Lambda_{c}^{+}$ modes it is a proton. Decay modes with oppositely charged leptons of the same lepton flavor are examples of flavorchanging neutral current (FCNC) processes, which are expected to be very rare because they cannot occur at tree level in the SM. Decay modes with two oppositely charged leptons of different flavor correspond to leptonflavor violating (LFV) decays and are essentially forbidden in the SM because they can occur only through lepton mixing. Decay modes with two leptons of the same charge are lepton-number violating (LNV) decays and are forbidden in the SM. Hence, decays of the form $X_{c}^{+} \rightarrow h^{ \pm} \ell^{\mp} \ell^{(\prime)+}$ provide sensitive tools to investigate physics beyond the SM. The most stringent existing upper limits [1-5] on the branching fractions for $X_{c}^{+} \rightarrow h^{ \pm} \ell^{\mp} \ell^{(1)+}$ decays range from 1 to $700 \times 10^{-6}$ and do not exist for most of the $\Lambda_{c}^{+}$decays.

FCNC processes have been studied extensively for $K$ and $B$ mesons, in $K^{0}-\bar{K}^{0}$ and $B^{0}-\bar{B}^{0}$ mixing, and in rare FCNC decays such as $s \rightarrow d \ell^{+} \ell^{-}, b \rightarrow s \gamma$, and $b \rightarrow$ $s \ell^{+} \ell^{-}$decays. The results agree with expectations within the framework of the SM [6]. There are ongoing efforts to improve the measurements and the theoretical predictions, and to measure new effects, such as $C P$ violation, in FCNC processes.

The recent observation of $D^{0}-\bar{D}^{0}$ mixing [7-9] has increased interest in FCNC processes in the charm sector. Of particular interest is the source of $D^{0}-\bar{D}^{0}$ mixing. If the mixing is due to physics beyond the SM, it could also give rise to measurable effects in FCNC charm decays. In the SM, the FCNC decays $X_{c}^{+} \rightarrow h^{+} \ell^{+} \ell^{-}$are expected to be heavily suppressed due to cancellations of amplitudes through the Glashow-Iliopoulos-Maiani (GIM) mechanism [10]. For example, the $c \rightarrow u \ell^{+} \ell^{-}$transitions illustrated in Fig. 1 yield branching fractions for $D \rightarrow X_{u} \ell^{+} \ell^{-}$of $\mathcal{O}\left(10^{-8}\right)[11,12]$. These decays are masked by the presence of long-distance contributions from intermediate vector resonances such as $D \rightarrow X_{u} V, V \rightarrow \ell^{+} \ell^{-}$, which are predicted to have branching fractions of $\mathcal{O}\left(10^{-6}\right)[11,12]$. The effect of these resonances can be separated from those due to short-range processes by applying selection criteria on the invariant mass of the $\ell^{+} \ell^{-}$pair. In radiative charm decays, $c \rightarrow u \gamma$, uncertainties in calculating the longdistance terms make it impossible to study the underlying short-distance physics [13].

The impact of several extensions of the SM on $D \rightarrow$ $X_{u} \ell^{+} \ell^{-}$decay rates has been estimated $[11,14,15]$; the largest effect not already ruled out is expected in certain $R$-parity violating supersymmetric models. Depending on the size of the $R$-parity violating couplings, branching fractions of up to $\mathcal{O}\left(10^{-5}\right)$ for different $D \rightarrow X_{u} \ell^{+} \ell^{-}$ decays are possible.

The only long-distance amplitudes relevant at the current experimental sensitivity are from $D^{+} \rightarrow \pi^{+} \phi$ and
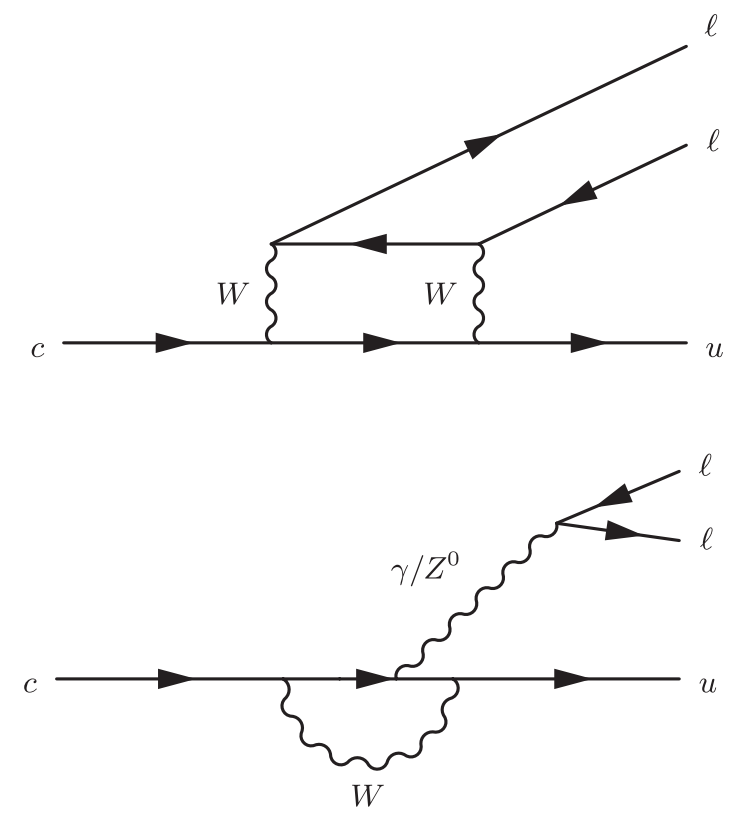

FIG. 1. Standard model short-distance contributions to the $c \rightarrow u \ell^{+} \ell^{-}$transition.

$D_{s}^{+} \rightarrow \pi^{+} \phi$ decays. The product of branching fractions, $\mathcal{B}\left(D_{(s)}^{+} \rightarrow \pi^{+} \phi\right) \mathcal{B}\left(\phi \rightarrow \ell^{+} \ell^{-}\right)$, is $\approx 2 \times 10^{-6}$ for $D^{+}$ and $\approx 1 \times 10^{-5}$ for $D_{s}^{+}$[16]. In this analysis, we measure the total decay rate of $D_{(s)}^{+} \rightarrow \pi^{+} \ell^{+} \ell^{-}$excluding the $\ell^{+} \ell^{-}$ mass region around the $\phi$ resonance.

\section{OVERVIEW}

The searches use data collected with the BABAR detector as described in Sec. III. Candidate decays are formed from two tracks identified as leptons $\left(\ell \ell^{(/)}\right)$and one track identified as a hadron $(h)$. Background events arise primarily from semileptonic $B$ and charm decays and from radiative QED events with converted photons. After applying basic kinematic selection criteria, we discriminate further between signal and background with a decay-modedependent likelihood ratio calculated from the measured momentum and flight length of the charm hadron candidate and the total energy detected in the event. The invariant mass distributions of the selected $h \ell \ell^{(\prime)}$ candidates are fit to extract the number of signal events, the number of combinatorial background events, and the number of background events due to nonleptonic charm decays with hadrons in the final state misidentified as leptons. Upper limits are determined for the decay rate to each final state with respect to the known decay rate for a hadronic threebody decay with similar kinematics so that many systematic uncertainties cancel in the ratio.

\section{THE BABAR DETECTOR AND DATA SET}

The BABAR detector was operated at the PEP-II asymmetric-energy storage rings at the SLAC National 
Accelerator Laboratory. The data sample comprises an integrated luminosity of $347 \mathrm{fb}^{-1}$ collected from $e^{+} e^{-}$ collisions at the $\mathrm{Y}(4 S)$ resonance and $37 \mathrm{fb}^{-1}$ collected $40 \mathrm{MeV}$ below the $\mathrm{Y}(4 S)$ resonance.

The BABAR detector is described in detail elsewhere [17]. The momenta of charged particles are measured with a combination of a five-layer silicon vertex tracker (SVT) and a 40-layer drift chamber (DCH), both in a $1.5 \mathrm{~T}$ magnetic field produced by a solenoid. The resolution of the transverse momentum $\left(p_{T}\right)$ is measured to be $\sigma\left(p_{T}\right) / p_{T}=0.0013\left(p_{T} / \mathrm{GeV} / c\right) \oplus 0.0045$. A detector of internally reflected Cherenkov radiation (DIRC) is used for charged particle identification. Pions, kaons, and protons are identified with likelihood ratios calculated from $d E / d x$ measurements in the SVT and DCH, and from the observed pattern of Cherenkov light in the DIRC. For the selection criteria used in this analysis, hadron identification efficiencies are approximately 98\%, 87\%, and $82 \%$ for pions, kaons, and protons, respectively. A finely segmented CsI (Tl) electromagnetic calorimeter (EMC) is used to detect and measure the energies of photons, and to identify electrons. For electrons, energy lost due bremsstrahlung is recovered from deposits in the EMC.

The EMC information, Cherenkov angle, and $d E / d x$ measurements are combined to define a likelihood ratio used to select electrons with a selection efficiency above $90 \%$ for electrons with a laboratory momentum above $0.5 \mathrm{GeV} / c$ and with less than $0.1 \%$ probability to misidentify a hadron as an electron. The instrumented flux return (IFR) contains resistive plate chambers and limited streamer tubes for muon and long-lived neutral-hadron identification. Variables characterizing track measurements in the IFR and the energy deposition in the EMC are combined in a neural network to select muon candidates. The muon identification efficiency is about $60 \%$ for muons with a laboratory momentum above $1.5 \mathrm{GeV} / c$, but decreases rapidly for lower momenta. The probability to misidentify a pion as a muon is about $1.5 \%$ for most of the relevant momentum range.

Event simulations are performed using the EVTGEN [18] Monte Carlo (MC) generator with a full detector simulation based on GEANT4 [19]. Signal and $\Lambda_{c}^{+} \rightarrow p K^{-} \pi^{+}$ MC events are generated with a three-body phase-space distribution, while $D_{(s)}^{+} \rightarrow \pi^{+} \phi$ MC events are generated with a Breit-Wigner resonance shape for the $\phi$ decay and an angular distribution appropriate for the $P$-wave $\phi$ decay. All signal events are simulated as $c \bar{c}$ continuum events. The distributions of the magnitudes of the charmhadron momenta measured in the $e^{+} e^{-}$center-of-mass frame $\left(p^{*}\right)$ are found to differ between simulated $c \bar{c}$ continuum events and data. In particular, the mean $p^{*}$ for $D_{s}^{+}$ mesons is lower in MC, while the mean $p^{*}$ for $\Lambda_{c}^{+}$baryons is higher. To correct for this, simulated $c \bar{c}$ events are weighted to yield the same $p^{*}$ distributions as those observed for the large samples of reconstructed charm decays in data used for normalization in Sec. V. The event weights lie between 0.6 and 1.8. Simulated events are also weighted to match the particle identification probabilities measured in control samples in data. Samples of simulated $c \bar{c}$ and $q \bar{q}$ $(q=u, d, s)$ continuum events and $B \bar{B}$ events, corresponding to 1.4 to 5 times the recorded data sample, are used to study background contributions.

\section{ANALYSIS PROCEDURE}

\section{A. Initial signal selection}

Charm hadron candidates are formed from one track identified as either a pion, kaon, or proton $(h)$ and two tracks, each of which is identified as an electron or a muon $\left(\ell \ell^{(\prime)}\right)$. The total charge of the three tracks is required to be \pm 1 . For three-track combinations with a pion or kaon track, the $h \ell \ell^{(\prime)}$ invariant mass is required to lie between 1.7 and $2.1 \mathrm{GeV} / c^{2}$; for combinations with a proton, the invariant mass is required to lie between 2.2 and $2.4 \mathrm{GeV} / c^{2}$.

The combinatorial background at low $p^{*}$ is very large and we therefore select charm hadron candidates with $p^{*}$ greater than $2.5 \mathrm{GeV} / c$. Because the $p^{*}$ of charm hadrons produced in $B$ decays is kinematically limited to be less than about $2.2 \mathrm{GeV} / c$, the signal candidates with $p^{*}$ greater than $2.5 \mathrm{GeV} / c$ are dominated by hadrons from continuum production. The main backgrounds remaining after this selection are QED events and semileptonic $B$ and charm decays, particularly events with two semileptonic decays.

The QED events are mainly radiative Bhabha, initialstate radiation, and two-photon events, which are all rich in electrons. These events are easily identified by their low multiplicity and/or highly jetlike topology. We strongly suppress this background by requiring at least five tracks in the event and that the hadron candidate be inconsistent with the electron hypothesis.

We suppress the background from semileptonic $B$ and charm decays by requiring the two leptons to be consistent with a common origin. This is achieved by requiring that the probability of the $\chi^{2}$ for the vertex fit be greater than 0.001 and that the distance of closest approach between the two lepton candidates be less than $250 \mu \mathrm{m}$.

For low $e^{+} e^{-}$invariant mass there is a significant background contribution from photon conversions and $\pi^{0}$ decays to $e^{+} e^{-} \gamma$. These are both removed by requiring $m\left(e^{+} e^{-}\right)>200 \mathrm{MeV} / c^{2}$.

For the $D_{(s)}^{+} \rightarrow \pi^{+} \ell^{+} \ell^{-}$decay modes, we exclude events with $0.95<m\left(e^{+} e^{-}\right)<1.05 \mathrm{GeV} / c^{2}$ and $0.99<$ $m\left(\mu^{+} \mu^{-}\right)<1.05 \mathrm{GeV} / c^{2}$ to reject decays through the $\phi$ resonance. The excluded regions for the two decay modes differ due to the larger radiative tail in the $\pi^{+} e^{+} e^{-}$decay mode. In order to perform cross-checks, we also select candidates for $D_{(s)}^{+} \rightarrow \pi \phi, \phi \rightarrow \ell^{+} \ell^{-}$decays by requiring $0.995 \mathrm{GeV} / c^{2}<m\left(e^{+} e^{-}\right)<1.030 \mathrm{GeV} / c^{2}$ or $1.005 \mathrm{GeV} /$ $c^{2}<m\left(\mu^{+} \mu^{-}\right)<1.030 \mathrm{GeV} / c^{2}$. 


\section{B. Likelihood selection}

After the initial event selection, significant combinatorial background contributions remain from semileptonic $B$ decays and other sources. These background sources are studied with samples of candidates in MC and in sidebands of the $h \ell \ell^{(1)}$ invariant mass distribution in data. The sidebands are defined to be $0.1 \mathrm{GeV} / c^{2}$ wide regions just below and above the signal regions defined above. The final candidate selection is performed by forming a likelihood ratio $R_{\mathcal{L}}$ and requiring the ratio to be greater than a minimum value $R_{\mathcal{L}}^{\mathrm{min}}$. The likelihood ratio is defined as

$$
R_{\mathcal{L}}(\vec{x})=\frac{\prod_{i} \mathcal{P}_{s, i}\left(x_{i}\right)}{\prod_{i} \mathcal{P}_{s, i}\left(x_{i}\right)+\prod_{i} \mathcal{P}_{b, i}\left(x_{i}\right)},
$$

where $x_{i}$ is the $i$ th discriminating variable, and $\mathcal{P}_{s, i}$ and $\mathcal{P}_{b, i}$ are the signal and background probability density functions (PDF) for the variable $x_{i}$. Correlations between variables are ignored in the likelihood ratio as they are found to be small. The likelihood ratio peaks near 1 for signal and near 0 for background. The signal PDFs are obtained by fitting the distribution of $x_{i}$ for simulated signal events, while the background PDFs are obtained from fits to the distribution of $x_{i}$ for candidates in the $h \ell \ell^{(\prime)}$ invariant-mass sidebands in data. The PDFs are defined by combinations of polynomial, Gaussian, and exponential functions found empirically to provide good descriptions of the signal MC and data sidebands. The parameter values in the PDFs are common across signal modes in which data or MC studies show the distributions to be consistent.

The following three discriminating variables are used in the likelihood ratio.

(i) Charm hadron candidate $p^{*}$.

The calculated $p^{*}$ for $h \ell \ell^{(/)}$candidates in which a lepton candidate is from a semileptonic $B$ decay can be larger than $2.2 \mathrm{GeV} / c$, but the distribution falls rapidly with increasing $p^{*}$.

(ii) Total reconstructed energy in the event.

Since neutrinos from semileptonic $B$ decays are not directly detected, the total observable energy in these events is less than the sum of the beam energies $(12 \mathrm{GeV})$. We calculate the total energy for each event as the sum of the energies of all reconstructed tracks (assuming each track to be a charged pion) and neutral EMC clusters.

(iii) Flight length significance.

The flight length significance is the ratio of the signed flight length to its uncertainty, where the signed flight length is the scalar product between the direction of the charm-hadron candidate and the position vector that points from the beam spot to the charm-hadron decay vertex. This variable has the most discrimination power for the long-lived $D^{+}$meson and the least for the shorter-lived $\Lambda_{c}^{+}$.
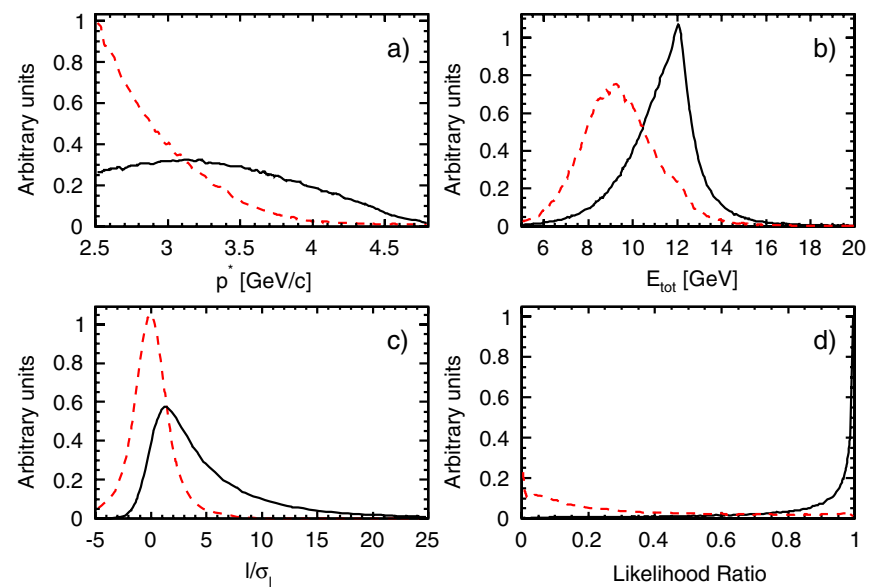

FIG. 2 (color online). Distributions of the three discriminating variables and the likelihood ratio, for $D^{+} \rightarrow \pi^{+} e^{+} e^{-}$candidates in signal MC (black solid curve) and in the $h \ell \ell^{(\prime)}$ invariant-mass sidebands from data (red dashed curve): (a) center-of-mass momentum $p^{*}$ of the charm-hadron candidate; (b) total energy in the event; (c) flight length significance; and (d) likelihood ratio [defined in Eq. (1)] calculated with the three discriminating variables. The signal and background distributions are normalized to the same area.

This variable is effective for suppressing non- $B$ combinatorial background.

Distributions of the three discriminating variables and of the likelihood ratio are shown in Fig. 2 for $D^{+} \rightarrow \pi^{+} e^{+} e^{-}$ candidates in $h \ell \ell^{(\prime)}$ invariant-mass sidebands (background) and in signal MC.

The minimum likelihood ratio value $R_{\mathcal{L}}^{\min }$ is chosen independently for each mode to provide the lowest expected upper limit on the branching ratio, as calculated from the simulated signal efficiency and the expected number of background events; the latter is estimated from the $h \ell \ell^{(\prime)}$ invariant-mass sidebands in data. In cases in which more than one candidate from the same event passes all selection criteria, including the likelihood-ratio requirement, the candidate with the highest $p^{*}$ is retained. The final signal selection efficiency lies between $0.5 \%$ and $7 \%$, depending on the signal mode.

\section{Fit procedure}

Extended, unbinned, maximum-likelihood fits are applied to the invariant-mass distributions for the $h^{ \pm} \ell^{\mp} \ell^{(1)+}$ candidates. The PDF we use for signal events is the so-called crystal ball function [20], which has an asymmetric component to describe the radiative tail in the mass distribution:

$$
\begin{aligned}
& P_{\mathrm{CB}}(m ; \mu, \sigma, \alpha, n) \\
& \quad= \begin{cases}e^{-(1 / 2)((m-\mu) / \sigma)^{2}} & \text { if } m \geq \mu-\alpha \sigma, \\
e^{-(1 / 2) \alpha^{2}}\left(\frac{n \sigma}{n \sigma^{-\alpha} \alpha^{2} \sigma-\alpha(m-\mu)}\right)^{n} & \text { if } m<\mu-\alpha \sigma .\end{cases}
\end{aligned}
$$


TABLE I. Branching fractions for the charm decays used for normalization [16].

\begin{tabular}{lr}
\hline \hline Decay mode & \multicolumn{1}{c}{ Branching fraction } \\
\hline$D^{+} \rightarrow \pi^{+} \phi_{K K}$ & $(2.72 \pm 0.13) \times 10^{-3}$ \\
$D_{s}^{+} \rightarrow \pi^{+} \phi_{K K}$ & $(2.32 \pm 0.14) \times 10^{-2}$ \\
$\Lambda_{c}^{+} \rightarrow p K^{-} \pi^{+}$ & $(5.0 \pm 1.3) \times 10^{-2}$ \\
\hline \hline
\end{tabular}

The four parameters $\mu, \sigma, \alpha$ and $n$ are determined from fits to signal $\mathrm{MC}$ candidates and are fixed to these values in the fits to data, with only the overall normalization as a free parameter. The width of the Gaussian component $(\sigma)$ is found to lie between 5 and $10 \mathrm{MeV} / c^{2}$, depending on the decay mode.

The invariant mass distributions of the combinatoric background events for the signal modes are described by first-order polynomials. The background slope is a free parameter in all fits.

An additional background arises from nonleptonic charm decays in which two hadrons are misidentified as leptons. This background component is almost negligible in the signal modes with electrons and is therefore only included in decay modes with two muons and for $D_{(s)}^{+} \rightarrow$ $K^{-} \mu^{+} e^{+}$, where there is a large $D^{+} \rightarrow K^{+} \pi^{+} \pi^{+}$background. The shape of this background is obtained from MC samples of major hadronic three-body charm decays. Each event is weighted according to the probability of misidentifying a pion as a lepton. The misidentification probability is measured from data as a function of momentum and angle with samples of $D^{0} \rightarrow K^{-} \pi^{+}$candidates. The misidentified nonleptonic charm decays are reconstructed at slightly lower $h^{+} \mu^{+} \mu^{-}\left(K^{-} \mu^{+} e^{+}\right)$mass than the signal events. The peak mass is shifted by about $15 \mathrm{MeV} / c^{2}$, which is sufficient separation from the signal peak for
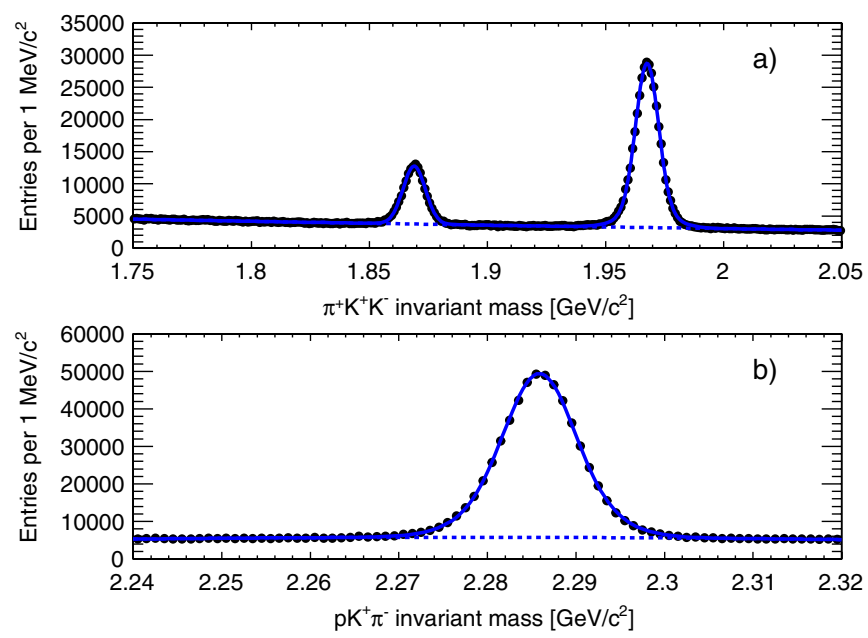

FIG. 3 (color online). Invariant mass distribution for (a) $\pi^{+} \phi_{K^{+} K^{-}}$and (b) $p K \pi$ candidates. The solid lines are the results of fits to double-Gaussian distributions for signals and a second-order polynomial for the background (dashed line).
TABLE II. Fitted signal yields for the normalization modes and signal efficiencies estimated from MC simulations. Only statistical uncertainties are quoted.

\begin{tabular}{lcc}
\hline \hline Decay mode & $N_{\text {sig }}$ & Efficiency \\
\hline$D^{+} \rightarrow \pi^{+} \phi_{K K}$ & $106800 \pm 500$ & $(15.44 \pm 0.07) \%$ \\
$D_{s}^{+} \rightarrow \pi^{+} \phi_{K K}$ & $338900 \pm 900$ & $(15.29 \pm 0.07) \%$ \\
$\Lambda_{c}^{+} \rightarrow p K^{-} \pi^{+}$ & $488700 \pm 2100$ & $(11.99 \pm 0.04) \%$ \\
\hline \hline
\end{tabular}

TABLE III. Summary of the multiplicative systematic uncertainties on the branching ratio for each signal decay mode (listed in the first column). In the second column, we give the fractional uncertainty due to the normalization mode. The next three columns list the fractional uncertainty due to the MC statistical error, uncertainties in particle identification efficiencies (PID), and in the likelihood-ratio efficiency (LR). The total systematic uncertainty, given in the final column, is the sum of the individual errors in quadrature.

\begin{tabular}{|c|c|c|c|c|c|}
\hline Decay mode & $\begin{array}{l}\text { Normalization } \\
\text { mode }\end{array}$ & $\begin{array}{c}\mathrm{MC} \\
\text { statistical } \\
\text { error }\end{array}$ & PID & LR & Total \\
\hline$D^{+} \rightarrow \pi^{+} e^{+} e^{-}$ & $2.6 \%$ & $1.8 \%$ & $2.3 \%$ & $9.8 \%$ & $10.6 \%$ \\
\hline$D^{+} \rightarrow \pi^{+} \mu^{+} \mu^{-}$ & $2.6 \%$ & $4.6 \%$ & $8.3 \%$ & $6.1 \%$ & $11.6 \%$ \\
\hline$D^{+} \rightarrow \pi^{+} e^{+} \mu^{-}$ & $2.6 \%$ & $3.1 \%$ & $4.4 \%$ & $9.8 \%$ & $11.5 \%$ \\
\hline$D^{+} \rightarrow \pi^{+} \mu^{+} e^{-}$ & $2.6 \%$ & $2.2 \%$ & $4.4 \%$ & $7.6 \%$ & $9.4 \%$ \\
\hline$D_{s}^{+} \rightarrow \pi^{+} e^{+} e^{-}$ & $2.1 \%$ & $1.1 \%$ & $2.3 \%$ & $0.9 \%$ & $3.4 \%$ \\
\hline$D_{s}^{+} \rightarrow \pi^{+} \mu^{+} \mu^{-}$ & $2.1 \%$ & $2.7 \%$ & $8.3 \%$ & $0.9 \%$ & $9.0 \%$ \\
\hline$D_{s}^{+} \rightarrow \pi^{+} e^{+} \mu^{-}$ & $2.1 \%$ & $2.6 \%$ & $4.4 \%$ & $2.9 \%$ & $6.2 \%$ \\
\hline$D_{s}^{+} \rightarrow \pi^{+} \mu^{+} e^{-}$ & $2.1 \%$ & $4.0 \%$ & $4.4 \%$ & $7.2 \%$ & $9.6 \%$ \\
\hline$D^{+} \rightarrow K^{+} e^{+} e^{-}$ & $2.6 \%$ & $1.4 \%$ & $2.8 \%$ & $5.5 \%$ & $6.8 \%$ \\
\hline$D^{+} \rightarrow K^{+} \mu^{+} \mu^{-}$ & $2.6 \%$ & $6.4 \%$ & $8.5 \%$ & $4.4 \%$ & $11.8 \%$ \\
\hline$D^{+} \rightarrow K^{+} e^{+} \mu^{-}$ & $2.6 \%$ & $2.9 \%$ & $4.7 \%$ & $5.8 \%$ & $8.4 \%$ \\
\hline$D^{+} \rightarrow K^{+} \mu^{+} e^{-}$ & $2.6 \%$ & $3.1 \%$ & $4.7 \%$ & $5.1 \%$ & $8.0 \%$ \\
\hline$D_{s}^{+} \rightarrow K^{+} e^{+} e^{-}$ & $2.1 \%$ & $1.5 \%$ & $2.8 \%$ & $2.0 \%$ & $4.3 \%$ \\
\hline$D_{s}^{+} \rightarrow K^{+} \mu^{+} \mu^{-}$ & $2.1 \%$ & $3.3 \%$ & $8.5 \%$ & $0.9 \%$ & $9.4 \%$ \\
\hline$D_{s}^{+} \rightarrow K^{+} e^{+} \mu^{-}$ & $2.1 \%$ & $2.2 \%$ & $4.7 \%$ & $2.0 \%$ & $5.9 \%$ \\
\hline$D_{s}^{+} \rightarrow K^{+} \mu^{+} e^{-}$ & $2.1 \%$ & $2.0 \%$ & $4.7 \%$ & $1.6 \%$ & $5.7 \%$ \\
\hline$\Lambda_{c}^{+} \rightarrow p e^{+} e^{-}$ & $3.4 \%$ & $1.4 \%$ & $2.0 \%$ & $5.4 \%$ & $6.8 \%$ \\
\hline$\Lambda_{c}^{+} \rightarrow p \mu^{+} \mu^{-}$ & $3.4 \%$ & $3.2 \%$ & $8.2 \%$ & $3.4 \%$ & $10.0 \%$ \\
\hline$\Lambda_{c}^{+} \rightarrow p e^{+} \mu^{-}$ & $3.4 \%$ & $3.4 \%$ & $4.3 \%$ & $5.6 \%$ & $8.5 \%$ \\
\hline$\Lambda_{c}^{+} \rightarrow p \mu^{+} e^{-}$ & $3.4 \%$ & $2.8 \%$ & $4.3 \%$ & $5.1 \%$ & $8.0 \%$ \\
\hline$D^{+} \rightarrow \pi^{-} e^{+} e^{+}$ & $2.6 \%$ & $1.3 \%$ & $2.3 \%$ & $5.7 \%$ & $6.8 \%$ \\
\hline$D^{+} \rightarrow \pi^{-} \mu^{+} \mu^{+}$ & $2.6 \%$ & $3.5 \%$ & $8.3 \%$ & $5.1 \%$ & $10.7 \%$ \\
\hline$D^{+} \rightarrow \pi^{-} \mu^{+} e^{+}$ & $2.6 \%$ & $1.6 \%$ & $4.4 \%$ & $4.6 \%$ & $7.1 \%$ \\
\hline$D_{s}^{+} \rightarrow \pi^{-} e^{+} e^{+}$ & $2.1 \%$ & $0.8 \%$ & $2.3 \%$ & $1.2 \%$ & $3.4 \%$ \\
\hline$D_{s}^{+} \rightarrow \pi^{-} \mu^{+} \mu^{+}$ & $2.1 \%$ & $2.2 \%$ & $8.3 \%$ & $1.2 \%$ & $8.9 \%$ \\
\hline$D_{s}^{+} \rightarrow \pi^{-} \mu^{+} e^{+}$ & $2.1 \%$ & $2.4 \%$ & $4.4 \%$ & $1.2 \%$ & $5.6 \%$ \\
\hline$D^{+} \rightarrow K^{-} e^{+} e^{+}$ & $2.6 \%$ & $1.4 \%$ & $2.8 \%$ & $5.7 \%$ & $7.0 \%$ \\
\hline$D^{+} \rightarrow K^{-} \mu^{+} \mu^{+}$ & $2.6 \%$ & $3.2 \%$ & $8.5 \%$ & $2.7 \%$ & $9.8 \%$ \\
\hline$D^{+} \rightarrow K^{-} \mu^{+} e^{+}$ & $2.6 \%$ & $2.4 \%$ & $4.7 \%$ & $3.9 \%$ & $7.1 \%$ \\
\hline$D_{s}^{+} \rightarrow K^{-} e^{+} e^{+}$ & $2.1 \%$ & $1.0 \%$ & $2.8 \%$ & $1.4 \%$ & $3.9 \%$ \\
\hline$D_{s}^{+} \rightarrow K^{-} \mu^{+} \mu^{+}$ & $2.1 \%$ & $2.8 \%$ & $8.5 \%$ & $0.6 \%$ & $9.2 \%$ \\
\hline$D_{s}^{+} \rightarrow K^{-} \mu^{+} e^{+}$ & $2.1 \%$ & $1.9 \%$ & $4.7 \%$ & $0.6 \%$ & $5.5 \%$ \\
\hline$\Lambda_{c}^{+} \rightarrow \bar{p} e^{+} e^{+}$ & $3.4 \%$ & $1.0 \%$ & $2.0 \%$ & $5.4 \%$ & $6.8 \%$ \\
\hline$\Lambda_{c}^{+} \rightarrow \bar{p} \mu^{+} \mu^{+}$ & $3.4 \%$ & $2.6 \%$ & $8.2 \%$ & $1.7 \%$ & $9.4 \%$ \\
\hline$\Lambda_{c}^{+} \rightarrow \bar{p} \mu^{+} e^{+}$ & $3.4 \%$ & $1.6 \%$ & $4.3 \%$ & $3.5 \%$ & $6.7 \%$ \\
\hline
\end{tabular}




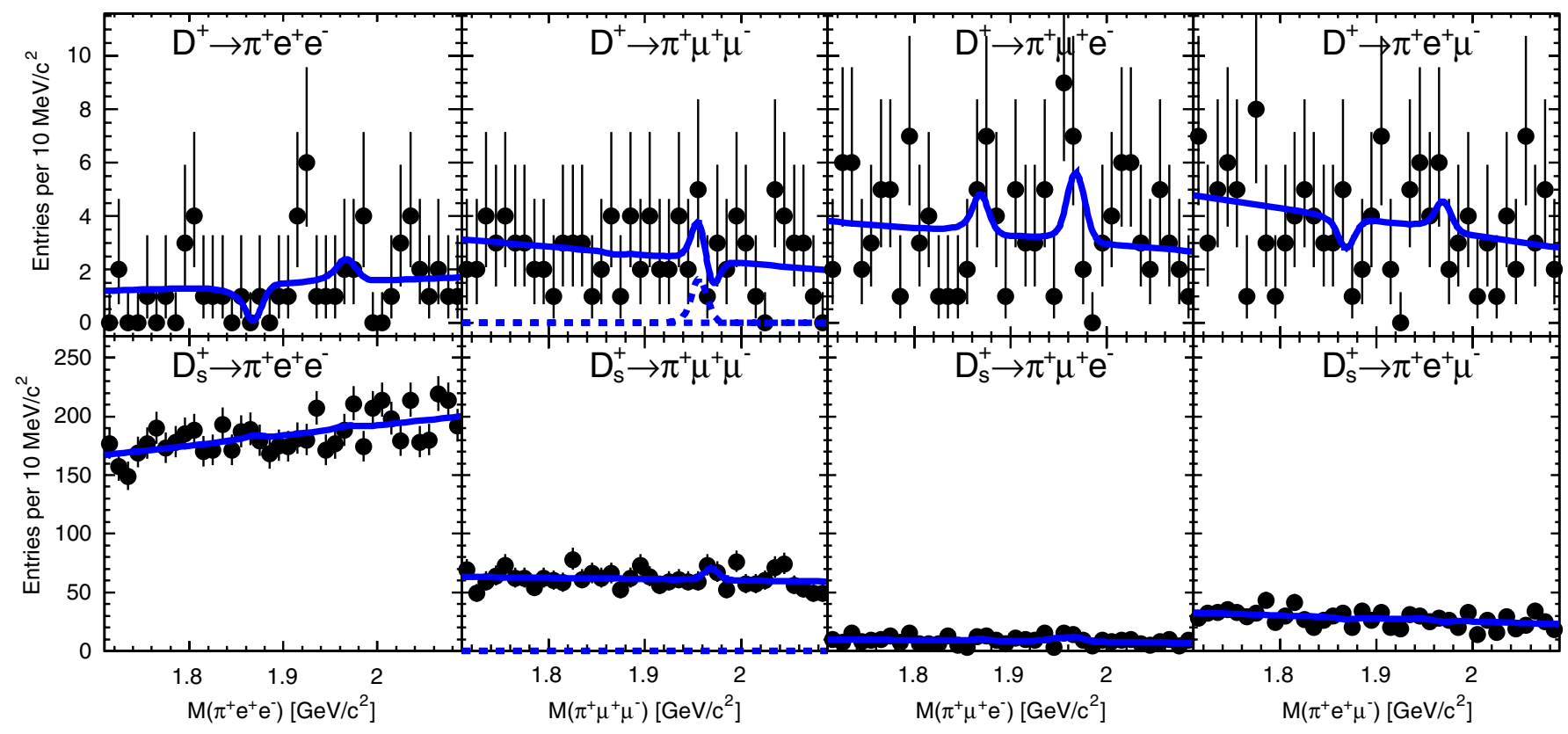

FIG. 4 (color online). Invariant-mass distributions for $D^{+} \rightarrow \pi^{+} \ell^{+} \ell^{(\prime)}-$ (top) and $D_{s}^{+} \rightarrow \pi^{+} \ell^{+} \ell^{(\prime)}-$ (bottom) candidates. The solid lines are the results of the fits. The background components for the dimuon modes in which muon candidates arise from misidentified hadrons are shown as dashed lines.

this background yield to be determined by the likelihood fit to data, without reliance on MC predictions of this yield.

\section{BRANCHING RATIO NORMALIZATION}

The measured signal yields are converted into branching ratios by normalizing them to the yields of known charm decays. We choose normalization modes with kinematics similar to the kinematics of the signal decays so that most systematic effects not related to particle identification cancel in the branching ratio. For the $D^{+}$and $D_{s}^{+}$mesons, we use decays to $\pi^{+} \phi$ as normalization modes, where the $\phi$ decays to $K^{+} K^{-}$. For the $\Lambda_{c}^{+}$, we use $\Lambda_{c}^{+} \rightarrow p K^{-} \pi^{+}$ as the normalization mode. The measured branching fractions for these modes are listed in Table I. We use the

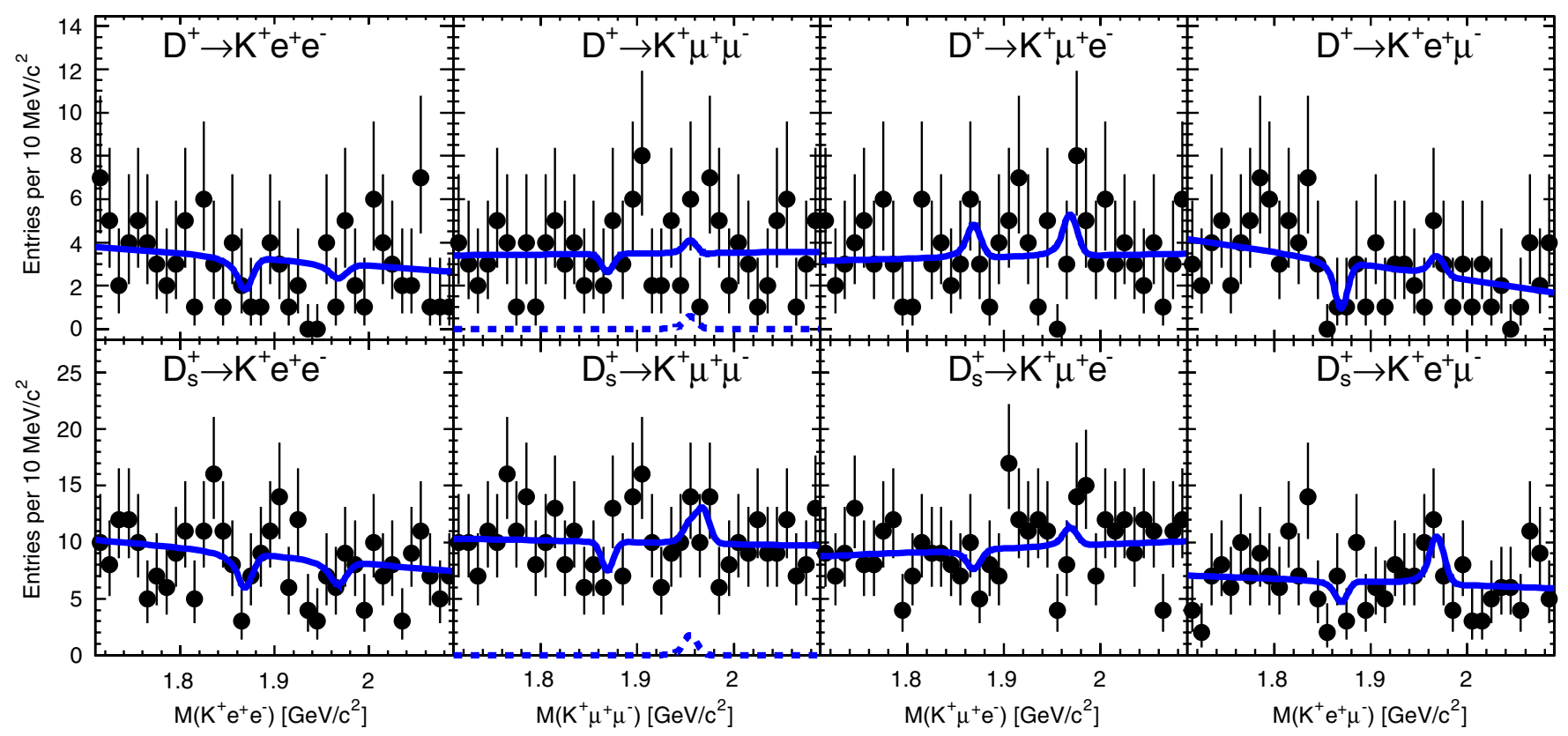

FIG. 5 (color online). Invariant-mass distributions for $D^{+} \rightarrow K^{+} \ell^{+} \ell^{(\prime)}-$ (top) and $D_{s}^{+} \rightarrow K^{+} \ell^{+} \ell^{(\prime)}-$ (bottom) candidates. The solid lines are the results of the fits. The background components for the dimuon modes in which muon candidates arise from misidentified hadrons are shown as dashed lines. 


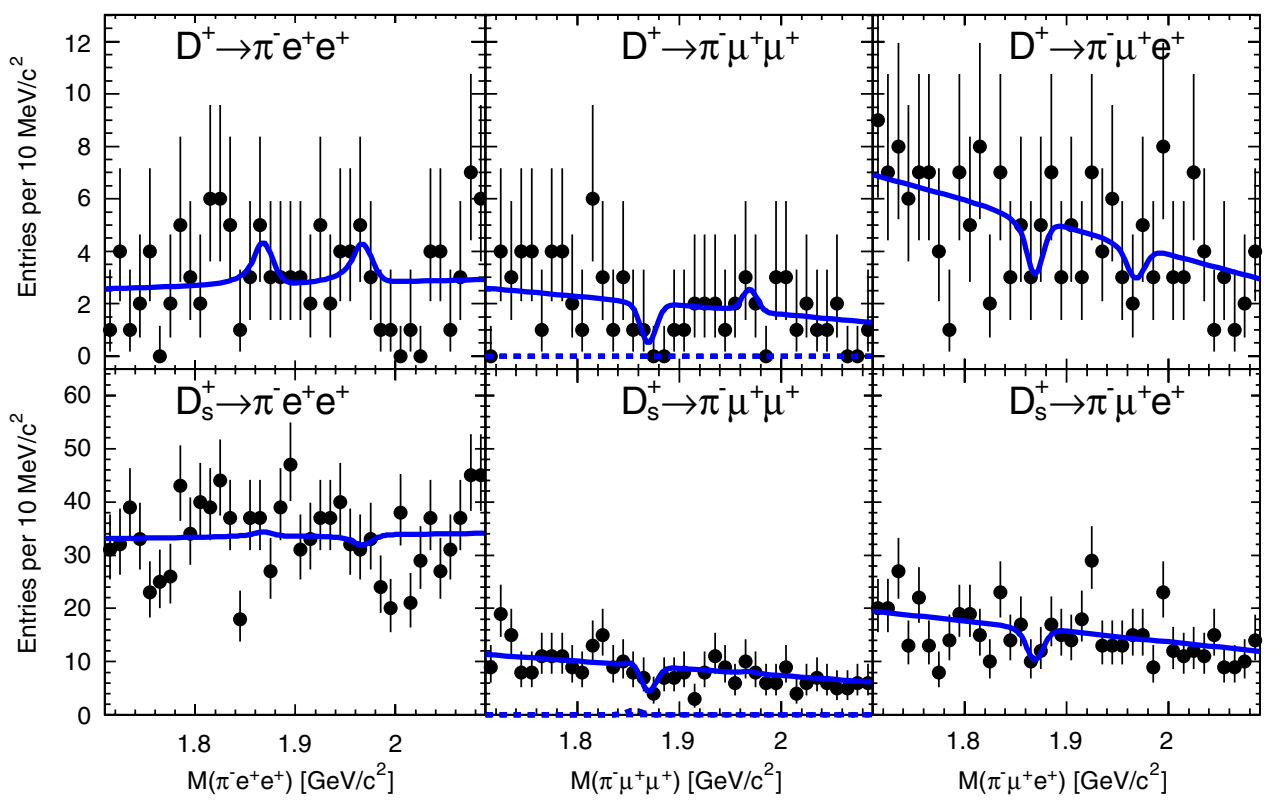

FIG. 6 (color online). Invariant-mass distributions for $D^{+} \rightarrow \pi^{-} \ell^{+} \ell^{(\prime)+}$ (top) and $D_{s}^{+} \rightarrow \pi^{-} \ell^{+} \ell^{(\prime)+}$ (bottom) candidates. The solid lines are the results of the fits. The background component for the dimuon modes in which muon candidates arise from misidentified hadrons is shown as a dashed line.

abbreviation $D_{(s)}^{+} \rightarrow \pi^{+} \phi_{K K}$ to denote $D_{(s)}^{+} \rightarrow \pi^{+} \phi, \phi \rightarrow$ $K^{+} K^{-}$decays

\section{A. Event selection}

The same selection criteria are applied for the normalization modes as the signal modes, except for the lepton identification and likelihood ratio requirements.
Instead, each daughter candidate in the $D_{(s)}^{+} \rightarrow \pi^{+} \phi_{K K}$ and $\Lambda_{c}^{+} \rightarrow p K^{-} \pi^{+}$decay modes is required to be consistent with the kaon, pion, or proton hypothesis, as appropriate. For the $D_{(s)}^{+} \rightarrow \pi^{+} \phi_{K K}$ decay modes, we further require the invariant mass of the kaon pair to lie within $15 \mathrm{MeV} / c^{2}$ of the world-average $\phi$ mass [16].

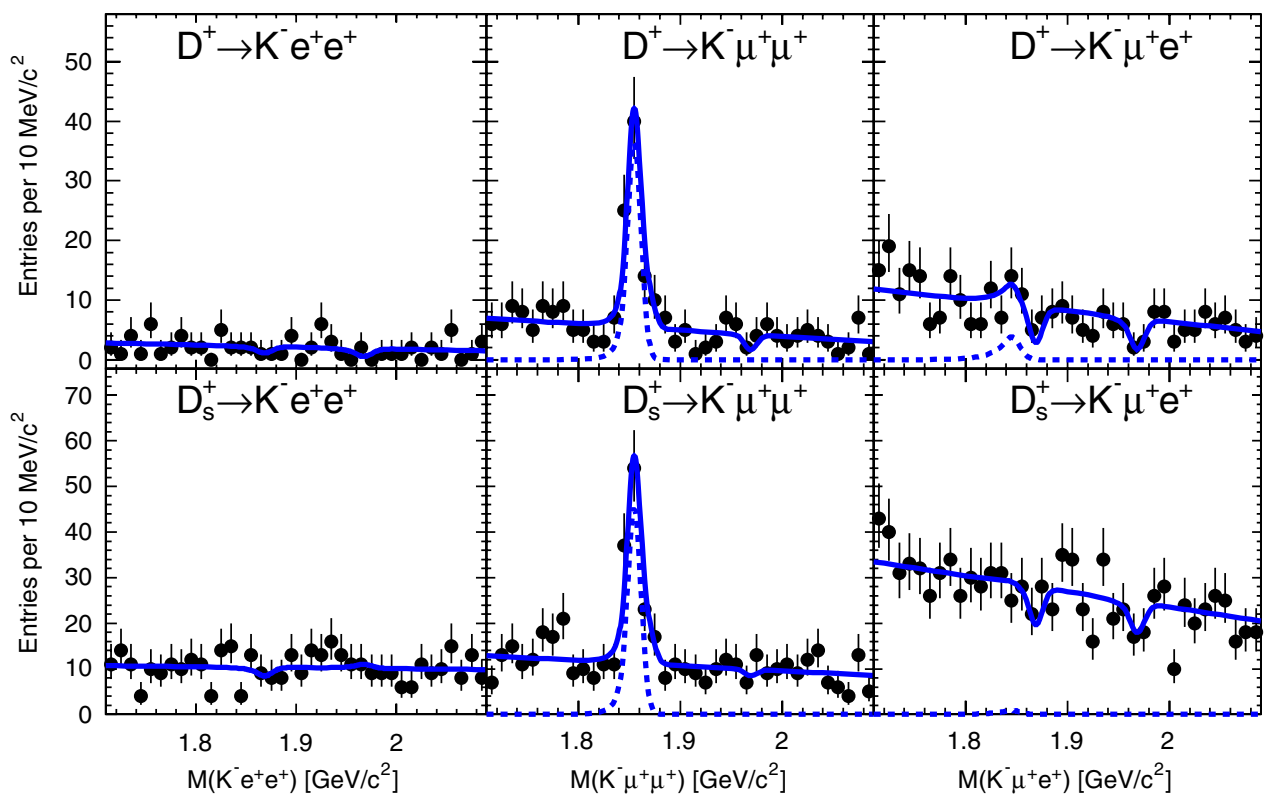

FIG. 7 (color online). Invariant-mass distributions for $D^{+} \rightarrow K^{-} \ell^{+} \ell^{(\prime)+}$ (top) and $D_{s}^{+} \rightarrow K^{-} \ell^{+} \ell^{(\prime)+}$ (bottom) candidates. The solid lines are the results of the fits. The background components for the dimuon modes and $D_{(s)}^{+} \rightarrow K^{-} \mu^{+} e^{+}$in which candidates arise from misidentified hadrons are shown as dashed lines. 


\section{B. Fit results}

For the normalization modes, radiative effects are negligible and we use the sum of two Gaussian distributions with a common mean to describe each of the $D^{+}, D_{s}^{+}$, and $\Lambda_{c}^{+}$signal invariant-mass distributions. All signal parameters are free in the fits to the normalization modes in data. The combinatorial background is described by a secondorder polynomial. The invariant-mass distributions for the normalization decay modes and the corresponding fit results are shown in Fig. 3. The fitted signal yields are listed in Table II, where we also list the efficiencies estimated from signal MC.

The $D_{(s)}^{+} \rightarrow \pi^{+} \phi_{K K}$ samples include a small component of nonresonant or $K^{+} K^{-} S$-wave $D_{(s)}^{+}$decays, while the branching fractions in Table I are extracted from decay amplitude analyses of Dalitz plot distributions for $D_{(s)}^{+} \rightarrow$ $\phi\left(K^{+} K^{-}\right) \pi^{+}$decays and therefore correspond only to the resonant component in which the $K^{+} K^{-}$are in a $P$-wave state. This component is estimated in our MC and data samples by projecting out the $P$-wave component by weighting each event with a factor that includes the reciprocal of its reconstruction efficiency and a normalized $L=2$ Legendre polynomial function of the $K^{+} K^{-}$helicity angle. The $P$-wave weighting removes virtually all background from Fig. 3, leaving only the $P$-wave components of $D^{+}$and $D_{(s)}^{+}$decays in their respective peak regions. The fractions of $D^{+}$and $D_{s}^{+}$decays found to proceed through $K^{+} K^{-} P$-waves are $94 \%$ and $93 \%$, respectively, and are used to correct the fit yields. For the $D_{s}^{+}$, this fraction is consistent with the $P$-wave yield found in a modelindependent study of the same $K^{+} K^{-}$invariant mass range. These fractions differ for other mass ranges, but are consistent with Ref. [21] within a $2 \%$ systematic uncertainty.

\section{SYSTEMATIC UNCERTAINTIES}

Most systematic effects are expected to cancel in the branching ratio since they affect both the signal and normalization modes. Therefore, the main systematic uncertainties arise from differences in selection, acceptance, and decay kinematics. Table III gives a summary of all systematic uncertainties related to the branching ratio calculation. An additional systematic uncertainty is associated with the estimate of the signal yield.

Systematic uncertainties related to the signal PDF parameters are investigated in two ways. First, the PDF parameters for data and $\mathrm{MC}$ are compared in the fits to the normalization modes. Differences can be due either to general data-MC tracking differences or to differences between the simulated charm hadron mass and the actual mass. Second, fits to the invariant mass of $J / \psi \rightarrow \ell^{+} \ell^{-}$ candidates from inclusive $B$ decays are compared between data and MC. The second comparison is sensitive to effects associated with lepton reconstruction and radiative tails. Based on these studies, the mean signal mass is varied by up to $2.5 \mathrm{MeV} / c^{2}$, depending on the decay mode. Based on the same studies, the widths of the signal PDFs are varied by $\pm 5 \%$. For the background shape assumption, the signal fits are repeated using a second-order polynomial as the background PDF instead of the nominal first-order

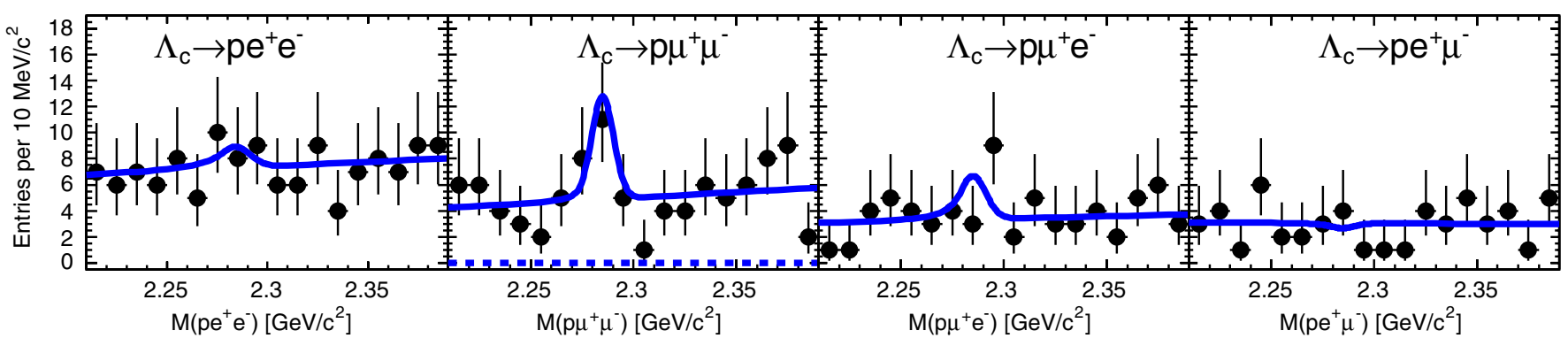

FIG. 8 (color online). Invariant-mass distributions for $\Lambda_{c}^{+} \rightarrow p \ell^{+} \ell^{(\prime)-}$ candidates. The solid lines are the results of the fits. The background component for the dimuon mode in which muon candidates arise from hadrons misidentified is shown as a dashed line.

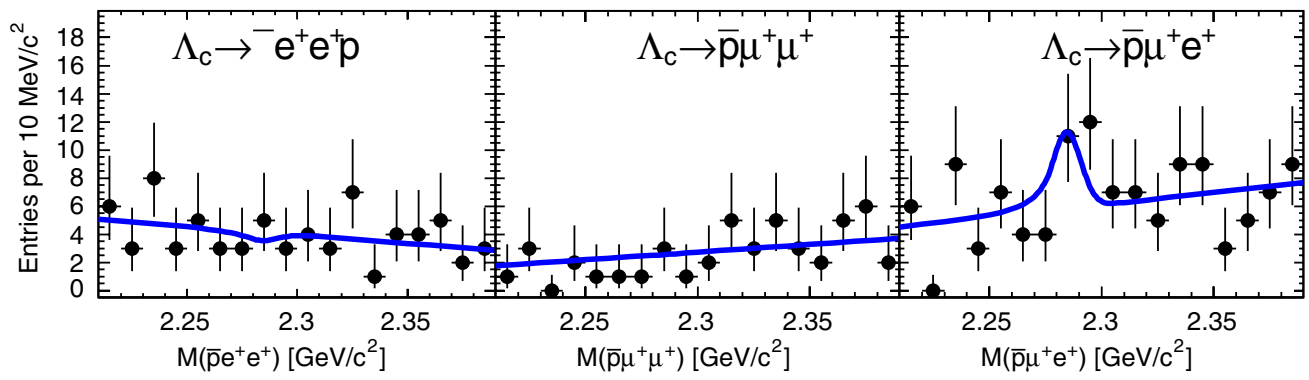

FIG. 9 (color online). Invariant-mass distributions for $\Lambda_{c}^{+} \rightarrow \bar{p} \ell^{+} \ell^{(/)+}$candidates. The solid lines are the results of the fits. 
polynomial. The PDF variation giving the worst upper limit on the branching ratio is used as an estimate of the systematic uncertainty.

For the normalization modes, the statistical uncertainties from the fits, the MC statistical errors, and uncertainties from the signal and background shapes are all about $1 \%$ or less. The main systematic uncertainty is due to intermediate resonances in the three-body decays. For the $D_{(s)}^{+}$ modes, where we select $K^{+} K^{-}$pairs consistent with the $\phi$ mass, this uncertainty is estimated by varying the $K^{+} K^{-}$ mass interval in order to alter the purity of the accepted $\phi$ candidates. For the $\Lambda_{c}^{+}$mode, the uncertainty is estimated from variations in the efficiency as a function of the $p K^{-}$ and $p \pi^{+}$invariant mass. For all three normalization modes the estimated uncertainty is approximately $2 \%$.

The particle identification efficiencies have associated systematic uncertainties of $0.5 \%$ for each pion, $0.7 \%$ for each kaon, $0.9 \%$ for each electron, and $4 \%$ for each muon. We do not evaluate a systematic uncertainty for the protons since, for the $\Lambda_{c}$ decays, both the signal and normalization

TABLE IV. Signal yields for the fits to the $35 X_{c}^{+} \rightarrow h^{ \pm} \ell^{\mp} \ell^{(\prime)+}$ event samples. The first error is the statistical uncertainty and the second is the systematic uncertainty. The third column lists the estimated signal efficiency. The fourth column gives for each signal mode the 90\% CL upper limit (UL) on the ratio of the branching fraction of the signal mode to that of the normalization mode (BR). The last column shows the $90 \%$ CL upper limit on the branching fraction for each signal mode (BF). The upper limits include all systematic uncertainties.

\begin{tabular}{|c|c|c|c|c|}
\hline Decay mode & Yield (events) & $\begin{array}{c}\text { Efficiency } \\
(\%)\end{array}$ & $\begin{array}{c}\text { BR UL, } \\
90 \% \text { CL }\left(10^{-4}\right)\end{array}$ & $\begin{array}{c}\text { BF UL, } \\
90 \% \text { CL }\left(10^{-6}\right)\end{array}$ \\
\hline$D^{+} \rightarrow \pi^{+} e^{+} e^{-}$ & $-3.9 \pm 1.6 \pm 1.7$ & 1.56 & 3.9 & 1.1 \\
\hline$D^{+} \rightarrow \pi^{+} \mu^{+} \mu^{-}$ & $-0.2 \pm 2.8 \pm 0.9$ & 0.46 & 24 & 6.5 \\
\hline$D^{+} \rightarrow \pi^{+} e^{+} \mu^{-}$ & $-2.9 \pm 3.4 \pm 2.4$ & 1.21 & 11 & 2.9 \\
\hline$D^{+} \rightarrow \pi^{+} \mu^{+} e^{-}$ & $3.6 \pm 4.3 \pm 1.3$ & 1.54 & 13 & 3.6 \\
\hline$D_{s}^{+} \rightarrow \pi^{+} e^{+} e^{-}$ & $8 \pm 34 \pm 8$ & 6.36 & 5.4 & 13 \\
\hline$D_{s}^{+} \rightarrow \pi^{+} \mu^{+} \mu^{-}$ & $20 \pm 15 \pm 4$ & 1.21 & 18 & 43 \\
\hline$D_{s}^{+} \rightarrow \pi^{+} e^{+} \mu^{-}$ & $-3 \pm 11 \pm 3$ & 2.16 & 4.9 & 12 \\
\hline$D_{s}^{+} \rightarrow \pi^{+} \mu^{+} e^{-}$ & $9.3 \pm 7.3 \pm 2.8$ & 1.50 & 8.4 & 20 \\
\hline$D^{+} \rightarrow K^{+} e^{+} e^{-}$ & $-3.7 \pm 2.9 \pm 3.3$ & 2.88 & 3.7 & 1.0 \\
\hline$D^{+} \rightarrow K^{+} \mu^{+} \mu^{-}$ & $-1.3 \pm 2.8 \pm 1.1$ & 0.65 & 16 & 4.3 \\
\hline$D^{+} \rightarrow K^{+} e^{+} \mu^{-}$ & $-4.3 \pm 1.8 \pm 0.6$ & 1.44 & 4.3 & 1.2 \\
\hline$D^{+} \rightarrow K^{+} \mu^{+} e^{-}$ & $3.2 \pm 3.8 \pm 1.2$ & 1.74 & 9.9 & 2.8 \\
\hline$D_{s}^{+} \rightarrow K^{+} e^{+} e^{-}$ & $-5.7 \pm 5.8 \pm 2.0$ & 3.20 & 1.6 & 3.7 \\
\hline$D_{s}^{+} \rightarrow K^{+} \mu^{+} \mu^{-}$ & $4.8 \pm 5.9 \pm 1.2$ & 0.85 & 9.1 & 21 \\
\hline$D_{s}^{+} \rightarrow K^{+} e^{+} \mu^{-}$ & $9.1 \pm 6.0 \pm 2.8$ & 1.74 & 5.7 & 14 \\
\hline$D_{s}^{+} \rightarrow K^{+} \mu^{+} e^{-}$ & $3.4 \pm 6.4 \pm 3.5$ & 2.08 & 4.2 & 9.7 \\
\hline$\Lambda_{c}^{+} \rightarrow p e^{+} e^{-}$ & $4.0 \pm 6.5 \pm 2.8$ & 5.52 & 0.8 & 5.5 \\
\hline$\Lambda_{c}^{+} \rightarrow p \mu^{+} \mu^{-}$ & $11.1 \pm 5.0 \pm 2.5$ & 0.86 & 6.4 & 44 \\
\hline$\Lambda_{c}^{+} \rightarrow p e^{+} \mu^{-}$ & $-0.7 \pm 2.9 \pm 0.9$ & 1.10 & 1.6 & 9.9 \\
\hline$\Lambda_{c}^{+} \rightarrow p \mu^{+} e^{-}$ & $6.2 \pm 4.6 \pm 1.8$ & 1.37 & 2.9 & 19 \\
\hline$D^{+} \rightarrow \pi^{-} e^{+} e^{+}$ & $4.7 \pm 4.7 \pm 0.5$ & 3.16 & 6.8 & 1.9 \\
\hline$D^{+} \rightarrow \pi^{-} \mu^{+} \mu^{+}$ & $-3.1 \pm 1.2 \pm 0.5$ & 0.70 & 7.5 & 2.0 \\
\hline$D^{+} \rightarrow \pi^{-} \mu^{+} e^{+}$ & $-5.1 \pm 4.2 \pm 2.0$ & 1.72 & 7.4 & 2.0 \\
\hline$D_{s}^{+} \rightarrow \pi^{-} e^{+} e^{+}$ & $-5.7 \pm 14 . \pm 3.4$ & 6.84 & 1.8 & 4.1 \\
\hline$D_{s}^{+} \rightarrow \pi^{-} \mu^{+} \mu^{+}$ & $0.6 \pm 5.1 \pm 2.7$ & 1.05 & 6.2 & 14 \\
\hline$D_{s}^{+} \rightarrow \pi^{-} \mu^{+} e^{+}$ & $-0.2 \pm 7.9 \pm 0.6$ & 2.23 & 3.6 & 8.4 \\
\hline$D^{+} \rightarrow K^{-} e^{+} e^{+}$ & $-2.8 \pm 2.4 \pm 0.2$ & 2.67 & 3.1 & 0.9 \\
\hline$D^{+} \rightarrow K^{-} \mu^{+} \mu^{+}$ & $7.2 \pm 5.4 \pm 1.6$ & 0.80 & 37 & 10 \\
\hline$D^{+} \rightarrow K^{-} \mu^{+} e^{+}$ & $-11.6 \pm 4.0 \pm 3.1$ & 1.52 & 6.8 & 1.9 \\
\hline$D_{s}^{+} \rightarrow K^{-} e^{+} e^{+}$ & $2.3 \pm 7.9 \pm 3.3$ & 4.10 & 2.1 & 5.2 \\
\hline$D_{s}^{+} \rightarrow K^{-} \mu^{+} \mu^{+}$ & $-2.3 \pm 5.0 \pm 2.8$ & 0.98 & 5.3 & 13 \\
\hline$D_{s}^{+} \rightarrow K^{-} \mu^{+} e^{+}$ & $-14.0 \pm 8.4 \pm 2.0$ & 2.26 & 2.4 & 6.1 \\
\hline$\Lambda_{c}^{+} \rightarrow \bar{p} e^{+} e^{+}$ & $-1.5 \pm 4.2 \pm 1.5$ & 5.14 & 0.4 & 2.7 \\
\hline$\Lambda_{c}^{+} \rightarrow \bar{p} \mu^{+} \mu^{+}$ & $-0.0 \pm 2.1 \pm 0.6$ & 0.94 & 1.4 & 9.4 \\
\hline$\Lambda_{c}^{+} \rightarrow \bar{p} \mu^{+} e^{+}$ & $10.1 \pm 5.8 \pm 3.5$ & 2.50 & 2.3 & 16 \\
\hline
\end{tabular}


modes contain a proton and the uncertainty therefore cancels. Uncertainties for particles of the same type are added linearly, while those for different types of particles are added in quadrature.

The systematic uncertainty on the efficiency of the likelihood-ratio requirement is estimated by applying the same likelihood-ratio selection to events in the normalization mode. The efficiency of this selection for the normalization-mode decays is not expected to be the same as for signal modes due to different kinematics. However, for the normalization modes, the efficiency can be measured for both data and for MC simulation; the difference is used as the systematic uncertainty. The largest variations are found for decay modes with the most stringent likelihood-ratio requirements.

In the calculation of the signal efficiency we assume that the decays populate three-body phase-space uniformly. The selection efficiency has some dependence on where the decay lies in the Dalitz plot. Ignoring the regions we explicitly remove in the selection, the efficiency varies by less than $25 \%$ around the average as a function of the $\ell^{\mp} \ell^{(\prime)+}$ invariant mass, with the lowest efficiency at low dilepton mass. This model dependence is not included in the systematic uncertainty.

\section{RESULTS}

The $h \ell \ell^{(/)}$invariant-mass distributions for signal candidates in all 35 decay modes are shown in Figs. 4-9. The signal yields obtained from the unbinned likelihood fits are listed in Table IV with statistical and systematic uncertainties. Only systematic uncertainties associated with the signal and background PDFs are included in the systematic uncertainty for the yields. The curves representing the fits are overlaid in the figures. The most significant signal is seen in the distribution for $\Lambda_{c}^{+} \rightarrow p \mu^{+} \mu^{-}$; the signal yield has a statistical-only significance of $2.6 \sigma$ as determined from the change in log-likelihood with respect to zero assumed signal events. With 35 different measurements, a $2.6 \sigma$ deviation is expected with about $25 \%$ probability.

We calculate upper limits on the branching ratios

$$
\begin{aligned}
& \mathcal{B}\left(D_{(s)}^{+} \rightarrow \pi^{ \pm} \ell^{\mp} \ell^{(\prime)+}\right) / \mathcal{B}\left(D_{(s)}^{+} \rightarrow \pi^{+} \phi\right), \\
& \mathcal{B}\left(D_{(s)}^{+} \rightarrow K^{ \pm} \ell^{\mp} \ell^{(\prime)+}\right) / \mathcal{B}\left(D_{(s)}^{+} \rightarrow \pi^{+} \phi\right), \quad \text { and } \\
& \mathcal{B}\left(\Lambda_{c}^{+} \rightarrow \stackrel{(-)}{p} \ell^{\mp} \ell^{(\prime)+}\right) / \mathcal{B}\left(\Lambda_{c}^{+} \rightarrow p K^{-} \pi^{+}\right)
\end{aligned}
$$

at $90 \%$ confidence level (CL). The upper limits are set using a Bayesian approach with a flat prior for the event yield in the physical region. The upper limit on the signal yield is defined as the number of signal events for which the integral of the likelihood from zero events to that number of events is $90 \%$ of the integral from zero to infinity. The systematic uncertainties are included in the likelihood as additional nuisance parameters. For comparison with previous measurements, the upper limits on the

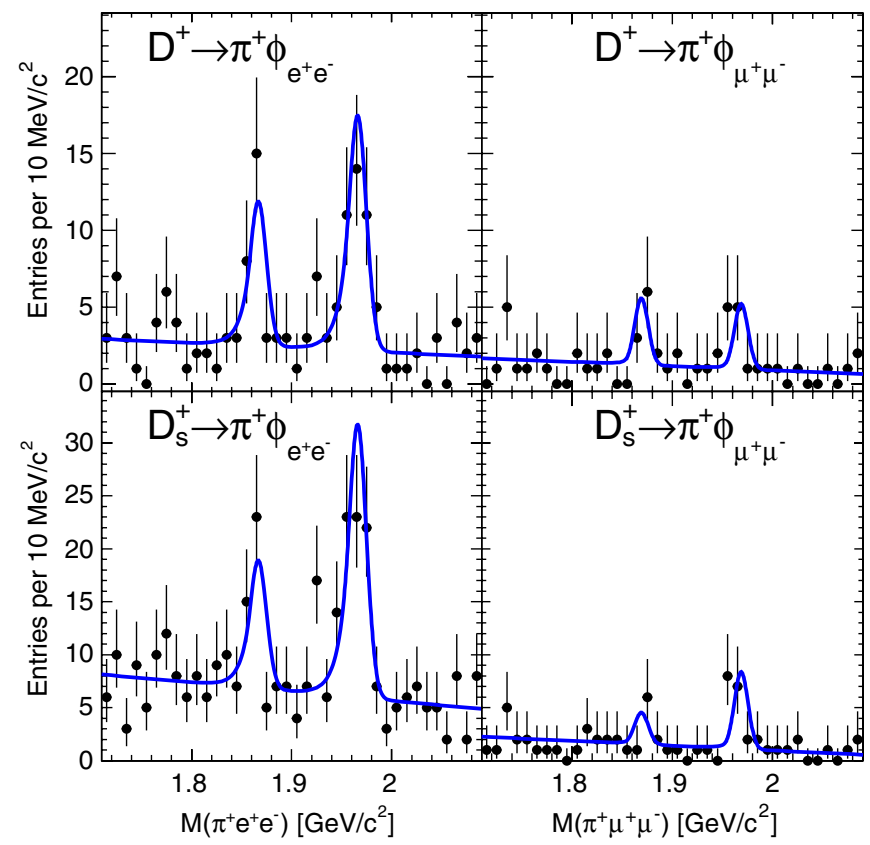

FIG. 10 (color online). Invariant-mass distribution for (left) $D_{(s)}^{+} \rightarrow \pi^{+} \phi_{e^{+} e^{-}}$and (right) $D_{(s)}^{+} \rightarrow \pi^{+} \phi_{\mu^{+} \mu^{-}}$candidates. Only events with dilepton invariant masses in the $\phi$ signal region defined in Sec. IVA are plotted. The solid lines are the results of the fits.

branching fractions at $90 \% \mathrm{CL}$, calculated using the data of Table I, are also given.

For 32 of the 35 decay modes, this analysis is more sensitive than existing measurements. In most cases, the improvement is significant (factor of 2 to 60). The largest improvements are seen for the LFV decays, which are all improved by at least a factor of 10 . The only $\Lambda_{c}^{+}$decay with a preexisting limit is $\Lambda_{c}^{+} \rightarrow p \mu^{+} \mu^{-}$, which we improve by roughly a factor of 8 . For all other $\Lambda_{c}^{+}$decays this paper presents the first limits. The only modes that do not provide a more sensitive limit are $D^{+} \rightarrow \pi^{-} e^{+} e^{+}, D^{+} \rightarrow$ $\pi^{+} \mu^{+} \mu^{-}$, and $D_{s}^{+} \rightarrow \pi^{+} \mu^{+} \mu^{-}$, where existing limits [1-3] are about a factor of 2 lower than those presented here.

As a cross-check, we also examine $D_{(s)}^{+} \rightarrow \pi^{+} \phi_{e^{+} e^{-}}$and $D_{(s)}^{+} \rightarrow \pi^{+} \phi_{\mu^{+} \mu^{-}}$events with dilepton invariant masses in

TABLE V. Signal yields for the fits to the $D_{(s)}^{+} \rightarrow \pi^{+} \phi_{\ell^{+} \ell^{-}}$ candidates. For the yields, the first uncertainty on the yield is statistical and the second is systematic. The last column shows the expected number of signal events, where the uncertainty is due to the systematic uncertainty assigned to the efficiency.

\begin{tabular}{lccc}
\hline \hline Decay mode & Yield (events) & $\begin{array}{c}\text { Efficiency } \\
(\%)\end{array}$ & $\begin{array}{c}\text { Expected } \\
\text { yield (events) }\end{array}$ \\
\hline$D^{+} \rightarrow \pi^{+} \phi_{e^{+} e^{-}}$ & $21.8 \pm 5.8 \pm 1.5$ & 5.65 & $22.2 \pm 1.1$ \\
$D^{+} \rightarrow \pi^{+} \phi_{\mu^{+} \mu^{-}}$ & $7.5 \pm 3.4 \pm 1.4$ & 1.11 & $4.5 \pm 0.4$ \\
$D_{s}^{+} \rightarrow \pi^{+} \phi_{e^{+} e^{-}}$ & $63 \pm 10 \pm 3$ & 6.46 & $79 \pm 3$ \\
$D_{s}^{+} \rightarrow \pi^{+} \phi_{\mu^{+} \mu^{-}}$ & $12.7 \pm 4.3 \pm 2.6$ & 1.07 & $13.1 \pm 1.2$ \\
\hline \hline
\end{tabular}


the $\phi$ signal region defined in Sec. IVA. The invariant mass distributions are shown in Fig. 10. Signals with a statistical significance of at least $3 \sigma$ are seen for all decays except for $D^{+} \rightarrow \pi^{+} \phi_{\mu^{+} \mu^{-}}$. The selection for $D^{+}$and $D_{s}^{+}$candidates differ through the likelihood-ratio criteria, which are optimized separately; however, signals for both hadrons can be seen with either selection. The mass distributions are therefore fit allowing for both a $D^{+}$and $D_{s}^{+}$signal, but only the signal yield for the hadron for which the likelihood ratio was constructed is used. Table $\mathrm{V}$ gives the fit yields. It also shows the expected yield assuming $\mathcal{B}\left(\phi \rightarrow \ell^{+} \ell^{-}\right)=$ $\mathcal{B}\left(\phi \rightarrow e^{+} e^{-}\right)=(2.95 \pm 0.03) \times 10^{-4}[16]$. The fit yields are in good agreement with expectations.

\section{CONCLUSIONS}

Searches for the decay modes $D_{(s)}^{+} \rightarrow \pi^{ \pm} \ell^{\mp} \ell^{(/)+}$, $D_{(s)}^{+} \rightarrow K^{ \pm} \ell^{\mp} \ell^{(1)+}$ and $\Lambda_{c}^{+} \rightarrow \stackrel{(-)}{p} \ell^{\mp} \ell^{(1)+}$ have been performed using $384 \mathrm{fb}^{-1}$ of $e^{+} e^{-}$annihilations collected with the $B A B A R$ detector. No signals are observed in the 35 modes investigated and we report upper limits between $0.4 \times 10^{-4}$ and $37 \times 10^{-4}$ at $90 \% \mathrm{CL}$ on the ratio of the branching fraction for the signal mode with respect to that of the normalization mode. This corresponds to limits on the branching fractions between $1 \times 10^{-6}$ and $44 \times 10^{-6}$. These limits are calculated under the assumption of threebody phase-space decays; the efficiency varies by up to $25 \%$ as a function of dilepton invariant mass. For 32 of the 35 decay modes studied, the limits are an improvement over the existing measurements and therefore provide more stringent constraints on physics beyond the SM.

\section{ACKNOWLEDGMENTS}

We are grateful for the extraordinary contributions of our PEP-II colleagues in achieving the excellent luminosity and machine conditions that have made this work possible. The success of this project also relies critically on the expertise and dedication of the computing organizations that support $B A B A R$. The collaborating institutions wish to thank SLAC for its support and the kind hospitality extended to them. This work is supported by the US Department of Energy and National Science Foundation, the Natural Sciences and Engineering Research Council (Canada), the Commissariat à l'Energie Atomique and Institut National de Physique Nucléaire et de Physique des Particules (France), the Bundesministerium für Bildung und Forschung and Deutsche Foschungsgemeinschaft (Germany), the Istituto Nazionale di Fisica Nucleare (Italy), the Foundation for Fundamental Research on Matter (The Netherlands), the Research Council of Norway, the Ministry of Education and Science of the Russian Federation, Ministerio de Ciencia e Innovación (Spain), and the Science and Technology Facilities Council (United Kingdom). Individuals have received support from the Marie-Curie IEF program (European Union), the A.P. Sloan Foundation (USA), and the Binational Science Foundation (USA-Israel).
[1] P. Rubin et al. (CLEO Collaboration), Phys. Rev. D 82, 092007 (2010).

[2] V. M. Abazov et al. (D0 Collaboration), Phys. Rev. Lett. 100, 101801 (2008).

[3] J. M. Link et al. (FOCUS Collaboration), Phys. Lett. B 572, 21 (2003).

[4] E. M. Aitala et al. (E791 Collaboration), Phys. Lett. B 462, 401 (1999).

[5] K. Kodama et al. (E653 Collaboration), Phys. Lett. B 345, 85 (1995).

[6] M. Antonelli et al., Phys. Rep. 494, 197 (2010).

[7] B. Aubert et al. (BABAR Collaboration), Phys. Rev. Lett. 98, 211802 (2007).

[8] M. Staric et al. (Belle Collaboration), Phys. Rev. Lett. 98, 211803 (2007).

[9] T. Aaltonen et al. (CDF Collaboration), Phys. Rev. Lett. 100, 121802 (2008).

[10] S. L. Glashow, J. Iliopoulos, and L. Maiani, Phys. Rev. D 2, 1285 (1970).

[11] G. Burdman, E. Golowich, J. Hewett, and S. Pakvasa, Phys. Rev. D 66, 014009 (2002).
[12] S. Fajfer, S. Prelovsek, and P. Singer, Phys. Rev. D 64, 114009 (2001).

[13] G. Burdman, E. Golowich, J. Hewett, and S. Pakvasa, Phys. Rev. D 52, 6383 (1995).

[14] S. Fajfer and S. Prelovsek, Phys. Rev. D 73, 054026 (2006).

[15] A. Paul, I. I. Bigi, and S. Recksiegel, Phys. Rev. D 83, 114006 (2011).

[16] K. Nakamura et al. (Particle Data Group), J. Phys. G 37, 075021 (2010).

[17] B. Aubert et al. (BABAR Collaboration), Nucl. Instrum. Methods Phys. Res., Sect. A 479, 1 (2002).

[18] D. J. Lange, Nucl. Instrum. Methods Phys. Res., Sect. A 462, 152 (2001).

[19] S. Agostinelli et al. (GEANT4 Collaboration), Nucl. Instrum. Methods Phys. Res., Sect. A 506, 250 (2003).

[20] M. J. Oreglia, Ph.D. thesis [Report No. SLAC-236, 1980], appendix D; J. E. Gaiser, Ph.D. thesis, [Report No. SLAC255, 1982], appendix E; T. Skwarnicki, Ph.D. thesis [Report No. DESY-F31-86-02, 1986], appendix E.

[21] P. del Amo Sanchez et al. (BABAR Collaboration), Phys. Rev. D 83, 052001 (2011). 